Revue des patrimoines

\title{
Le patrimoine rural en Languedoc-Roussillon : acquis et perspectives du travail d'inventaire
}

\section{Marie-Sylvie Grandjouan}

\section{(2) OpenEdition}

\section{Journals}

Édition électronique

URL : http://journals.openedition.org/insitu/2325

DOI : 10.4000/insitu.2325

ISSN : 1630-7305

Éditeur

Ministère de la Culture

Référence électronique

Marie-Sylvie Grandjouan, «Le patrimoine rural en Languedoc-Roussillon : acquis et perspectives du travail d'inventaire », In Situ [En ligne], 5 | 2004, mis en ligne le 19 avril 2012, consulté le 14 novembre 2019. URL : http://journals.openedition.org/insitu/2325; DOI : 10.4000/insitu.2325

Ce document a été généré automatiquement le 14 novembre 2019.

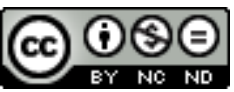

In Situ Revues des patrimoines est mis à disposition selon les termes de la licence Creative Commons Attribution - Pas d'Utilisation Commerciale - Pas de Modification 4.0 International. 


\title{
Le patrimoine rural en Languedoc- Roussillon : acquis et perspectives du travail d'inventaire
}

\author{
Marie-Sylvie Grandjouan
}

1 Parallèlement à l'étude de la ville de Montpellier, vingt cantons ruraux ont été inventoriés par le Service régional de l'Inventaire de Languedoc-Roussillon; ils se répartissent du sud au nord de la région, de la côte méditerranéenne jusqu'aux confins de l'Auvergne.

2 La masse d'informations accumulée est considérable mais hétérogène. Du fait de l'ancienneté du service, elle se ressent des aléas d'une méthode en continuelle évolution. Les résultats des premiers travaux d'inventaire réalisés dans le département des Pyrénées-Orientales en 1967 sont révélateurs des pôles d'intérêt de chercheurs qui, formés à l'histoire de l'art, étaient peu préparés à l'exploration de l'ensemble du patrimoine d'un territoire et l'on ne s'étonnera pas que l'architecture religieuse et les monuments civils les plus prestigieux aient été privilégiés. Ainsi, dans ce département, seules six maisons de village furent alors étudiées, et aucune ferme. Nous ne tiendrons compte ici que des travaux qui ont été menés à terme, c'est-à-dire jusqu'à la mise en forme des dossiers et à l'intégration des notices dans la base Mérimée du ministère de la Culture, en particulier les territoires pour lesquels les notices comprennent des champs descriptifs et historiques relativement développés et ceux qui ont fait l'objet de synthèses sous forme d'observations générales ou de publications.

3 Sur la totalité des notices enregistrées dans la base Mérimée (la ville de Montpellier mise à part), nous pouvons aujourd'hui dégager un corpus de 1100 unités comprenant des maisons de village, des fermes (421) et des habitations temporaires comme les cabanes ou les mazets. Leur répartition du sud au nord de la région constitue un échantillonnage de l'habitat "vernaculaire" des zones géographiques suivantes: la plaine littorale comprenant la zone des étangs et la grande plaine viticole, la moyenne vallée de l'Hérault, une partie des Grands Causses (Larzac méridional, causse de Sauveterre) et tout au nord de la région, aux confins de l'Auvergne, un territoire 
appartenant à la Margeride. On peut encore citer un canton à la géographie complexe, à l'est du département de la Lozère, entre le mont Lozère granitique et les schistes de l'Ardèche.

Quel que soit le type des enquêtes de terrain, préinventaire ou inventaire topographique ${ }^{1}$, les observations ont été faites à l'échelle de la parcelle, à l'aide du plan cadastral pour les villages et les écarts et de la carte I.G.N. au 1/25000 pour le repérage des édifices isolés. Les notes de terrain, plans cadastraux commentés, croquis consignés sur des carnets, ont permis de dégager les caractères architecturaux (structures, matériaux), de décrire les éléments de décor, de faire la liste des dates inscrites, d'aboutir à la rédaction d'observations générales et enfin à la sélection d'édifices à étudier; après la réalisation de ces synthèses, illustrées de cartes et de tableaux et donnant le dénombrement des édifices repérés, cette masse d'information n'a pas été conservée. Deux essais "d'information" du repérage ont cependant été réalisés en Lozère avec la constitution de fiches d'analyse de l'architecture des maisons ou des fermes. Dans le canton de Chanac ces fiches manuscrites ont permis un premier comptage d'éléments de typologie (porche surélevé, galerie haute, ouverture des cours, etc.). Pour l'inventaire des cantons de Saint-Alban et du Malzieu, nous avons informatisé cette fiche, après une adaptation nécessaire à un nouveau territoire ${ }^{2}$.

\section{Quelques exemples}

\section{La plaine littorale}

La plaine qui s'étend depuis le delta du Rhône jusqu'aux Pyrénées a été le cadre des premiers travaux du service qui portèrent sur des cantons littoraux: Rivesaltes et Saint-Laurent de la Salanque dans le département des Pyrénées-Orientales (1967), Coursan et Sigean dans l'Aude (1969). Dans le Gard, le canton d'Aigues-Mortes fut étudié à partir de 1967 et les résultats publiés en 1973 dans la collection des Inventaires topographiques ${ }^{3}$; enfin, dans l'Hérault, le canton de Mauguio.

Dans le canton d'Aigues-Mortes, il y avait en 1973 une centaine de mas dont seulement quinze sont indiqués au XVIII siècle sur la carte de Cassini. Leur présence dans cette zone couverte d'étangs et de marécages témoigne de la mise en culture de nouvelles terres grâce aux drainages réalisés dans le courant des XVII ${ }^{\mathrm{e}}$ et $\mathrm{XVIII}^{\mathrm{e}}$ siècles pour introduire une production céréalière ${ }^{4}$. Les sept édifices sélectionnés appartiennent tous à cette période. Le respect de la méthode de l'Inventaire, qui imposait alors une limite chronologique impérative, nous conduisit à laisser de côté les édifices postérieurs à 1850. Les grands domaines de la fin du XIXe siècle et leurs imposants celliers dits “caves", dont l'expansion correspond à la découverte du rôle de l'inondation des vignes dans la lutte contre le phylloxéra, ont donc été délibérément écartés (fig. $\left.\mathbf{n}^{\circ} \mathbf{1}\right)$. 
Figure 1

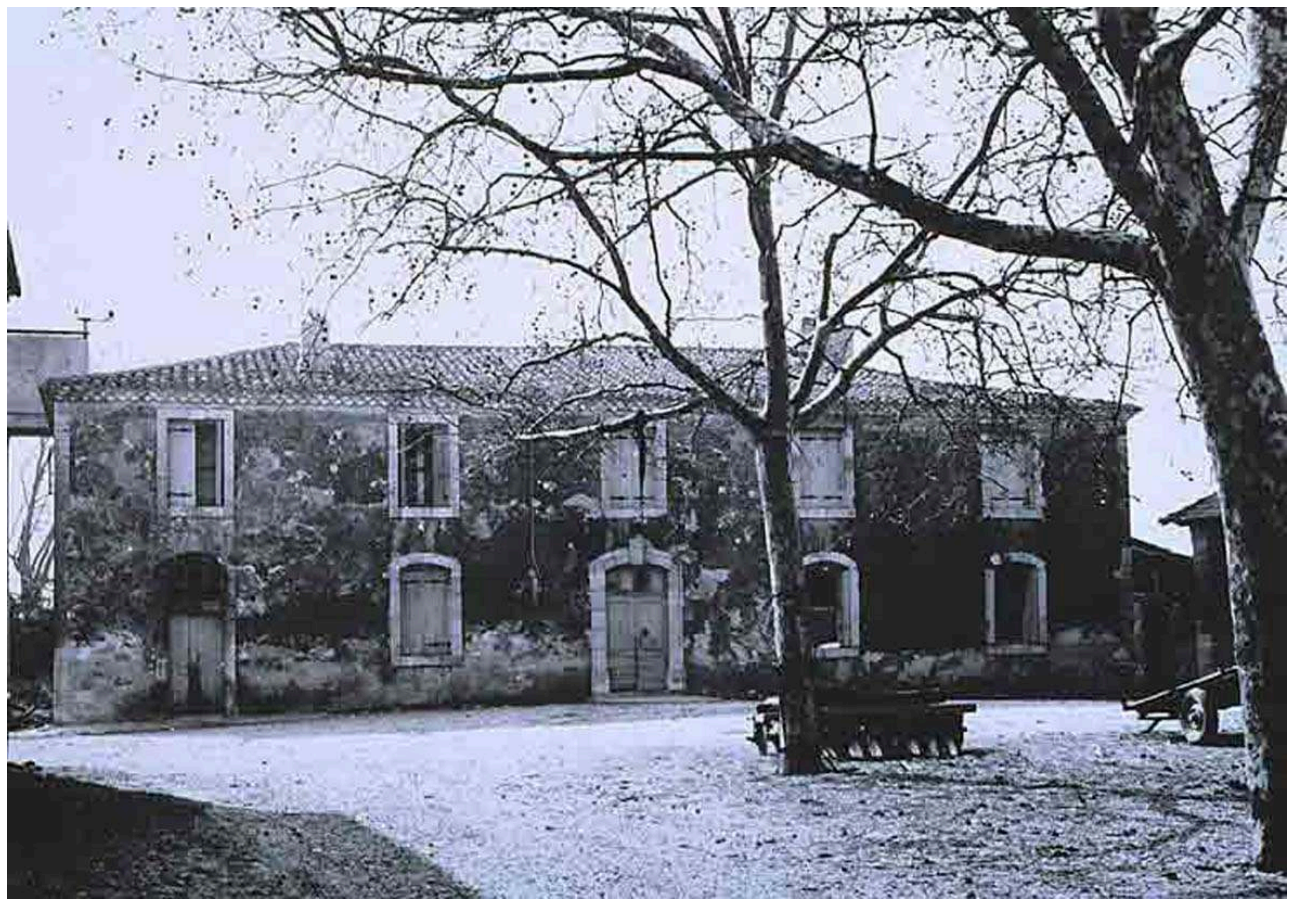

Mas de Quincandon (Aigues-Mortes). Vue d'ensemble du logis. Ce bâtiment de plan rectangulaire, couvert d'un toit à croupes, se compose d'un rez-de-chaussée de plain-pied et d'un vaste comble à surcroît

Phot. Inv. J.C. Jacques @ Inventaire général, ADAGP, 1994

Figure 2

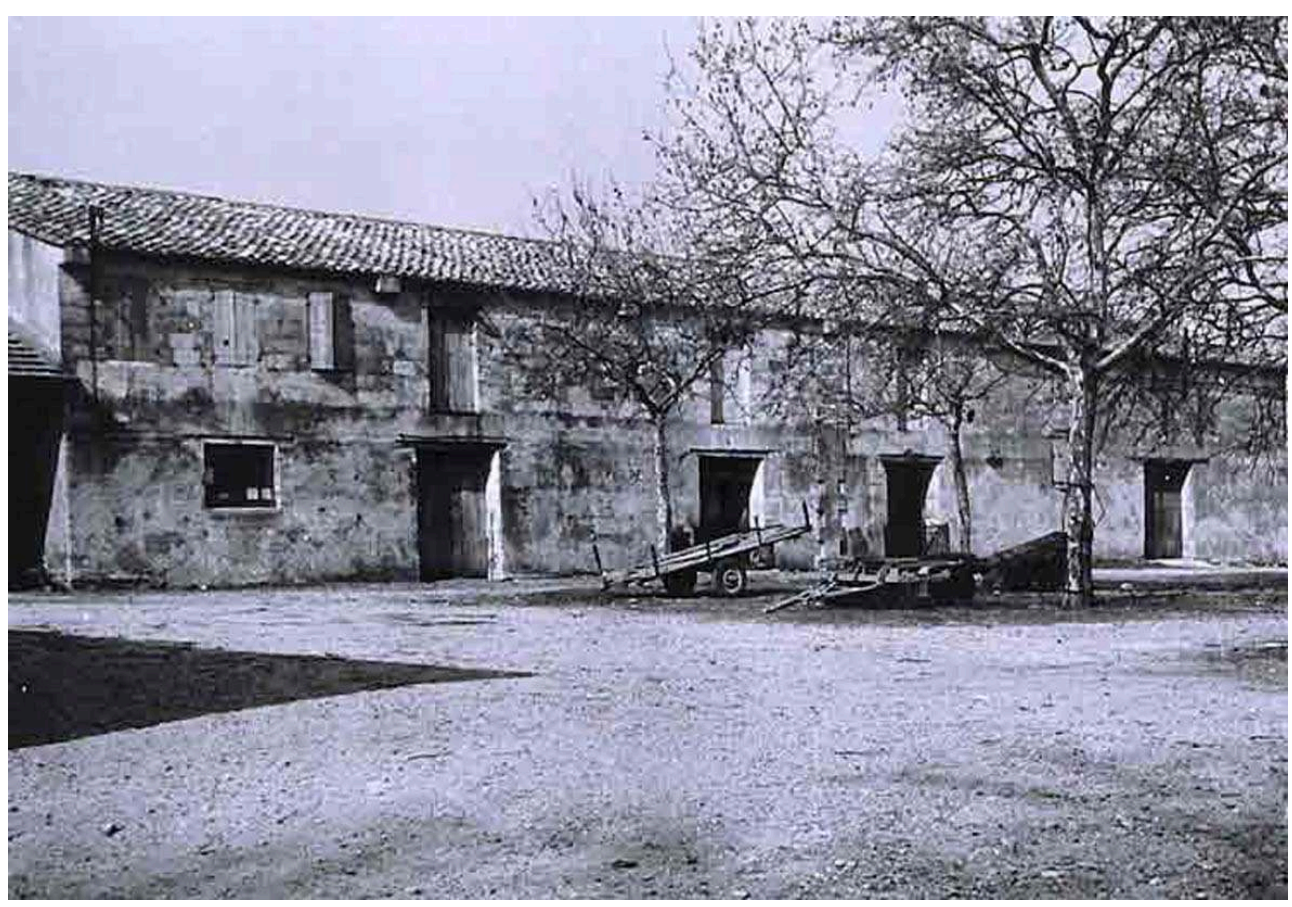

Mas de Quincandon (Aigues-Mortes). Les communs

Phot. Inv. J.C. Jacques () Inventaire général, ADAGP, 1994 


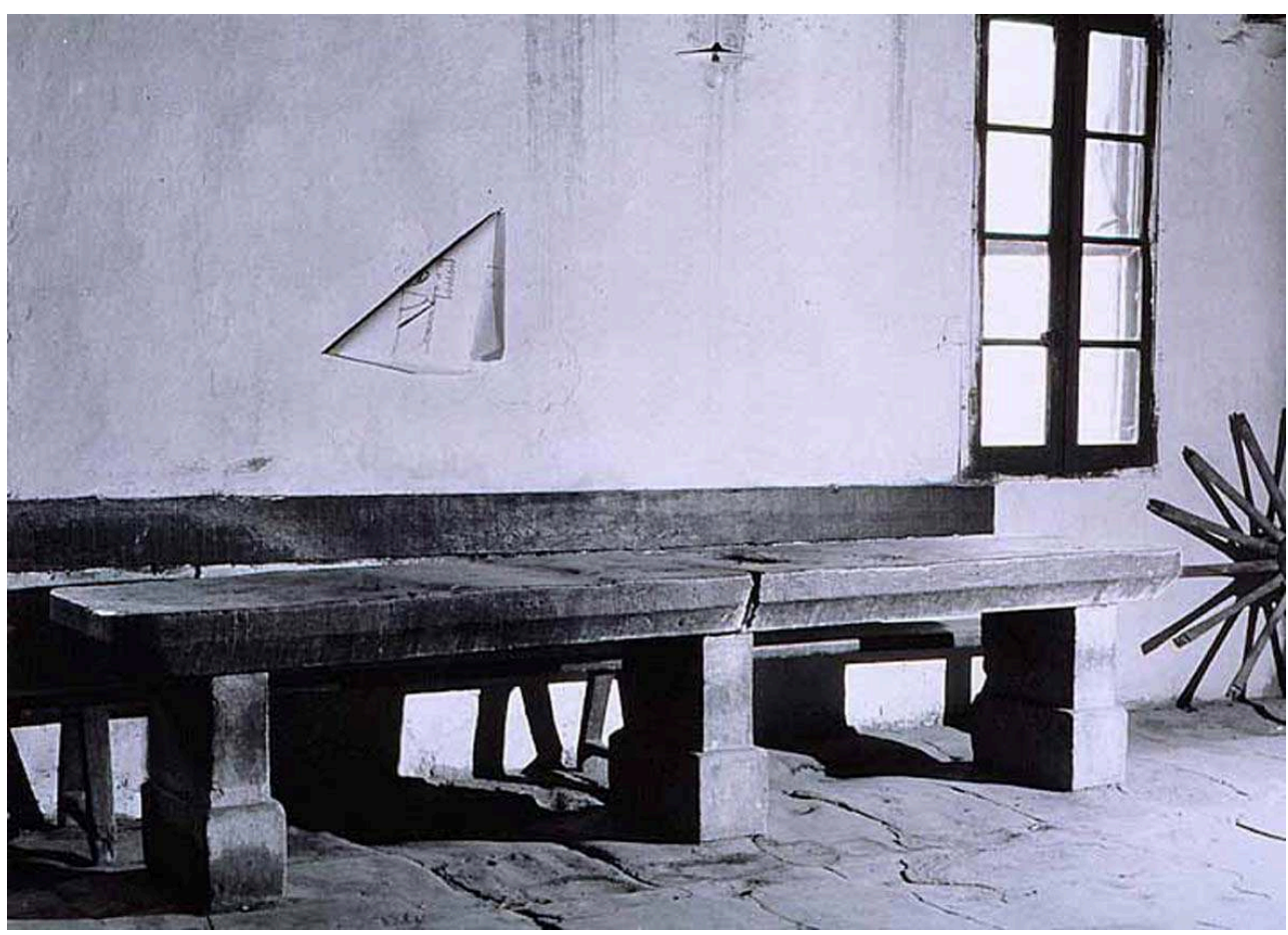

Mas de Quincandon (Aigues-Mortes). La table en pierre de la cuisine

Phot. Inv. J.C. Jacques @ Inventaire général, ADAGP, 1994

7 Le mas de Quincandon, implanté sur la commune d'Aigues-Mortes, date probablement de la fin du XVIII siècle. Isolé au milieu d'un grand domaine, il présentait en 1972 trois bâtiments disjoints disposés en $\mathrm{U}$ de façon symétrique par rapport à l'axe du logis : d'un côté les communs avec les logements des ouvriers, la forge et un hangar, l'autre côté est entièrement occupé par le grand bâtiment de la cave (fig. $\mathbf{n}^{\circ}{ }^{2}$ ) construit à la fin du XIX ${ }^{e}$ siècle.

Cette dernière construction marque la transformation que connaît alors l'agriculture, la vigne se substituant aux céréales (fig. $\mathbf{n}^{\circ} \mathbf{3}$ ).

Dans le cadre de l'enquête réalisée dans le canton de Mauguio (Hérault), les caractères généraux des grandes fermes ou " mas" de la fin du XIX siècle $^{5}$ ont été étudiés à partir des bâtiments présents sur le territoire du chef-lieu. Ce sont des fermes isolées au milieu de vastes domaines d'ancienne origine qui appartenaient à de riches familles montpelliéraines. En 1972, on pouvait encore identifier les fonctions des différents bâtiments qui témoignaient de l'évolution de l'agriculture depuis la fin du XVIII ${ }^{\mathrm{e}}$ siècle avec le passage, peu après 1850, d'une polyculture associant les céréales et l'élevage (moutons et porcs) à la monoculture de la vigne. Ces mas sont de grandes dimensions. Les différents bâtiments, écurie, bergeries, granges, porcherie, pigeonnier, logements pour les ouvriers, sont disposés sur trois des côtés de la cour fermée. Par son importance et son décor, la maison de maître évoque le "château" des domaines édifiés par les grands producteurs de vin à la fin du siècle dans le Biterrois ou la plaine audoise. Le bâtiment le plus vaste est toujours le cellier dont les dimensions expriment l'importance du domaine.

Le mas de Guillermain représente le cas extrême d'un établissement entièrement reconstruit sur les plans d'un architecte, Ch. Marcadier, en 1889, fait exceptionnel qui 
illustre la tendance à introduire dans les constructions rurales le savoir-faire des architectes et des ingénieurs. C'est le type même de "l'usine à vin " qui se caractérise par l'importance des bâtiments d'exploitation, en particulier de la "cave" qui abrite les foudres et le matériel de vinification, et des communs (fig. $\mathbf{n}^{\circ} \mathbf{4}$ ).

11 Ces grands mas possèdent tous une maison de maître dont les caractères architecturaux relèvent du style éclectique et pompeux de la fin du XIX ${ }^{e}$ siècle, contrastant avec les bâtiments d'exploitation qui gardent les caractères de l'architecture traditionnelle (fig. $n^{\circ} 5,6,7$ ).

Figure 4

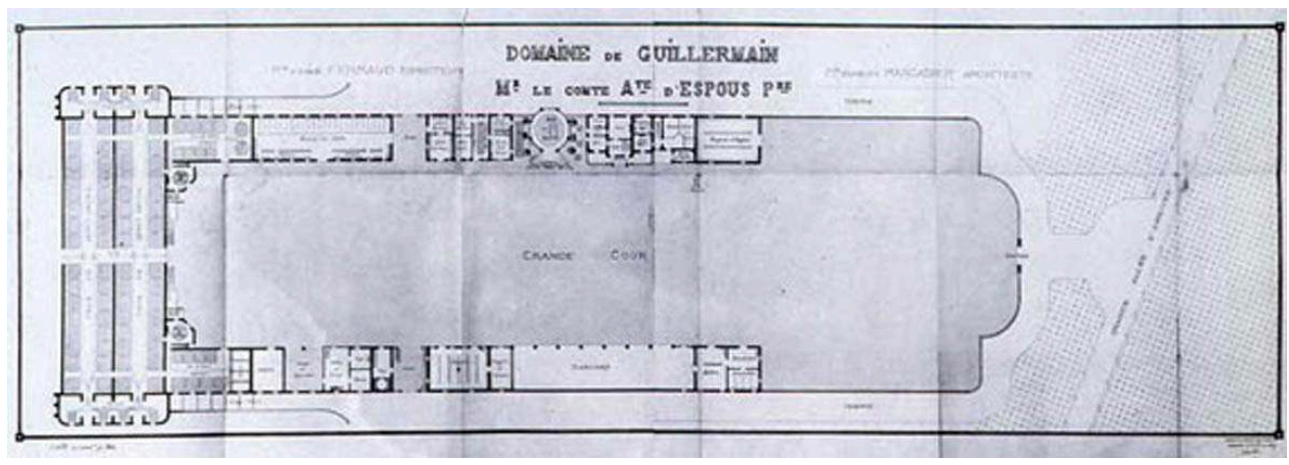

Mas de Guillermain (Mauguio). Domaine de Guillermain, $\mathrm{M}^{\mathrm{r}}$ le comte $\mathrm{A}^{\text {te }} \mathrm{d}^{\prime}$ Espous. Pre. Pierre Fermaud, directeur. Charles Marcadier, architecte. Plan d'ensemble

Phot. Inv. J.C. Jacques @ Inventaire général, ADAGP, 1994

Figure 5

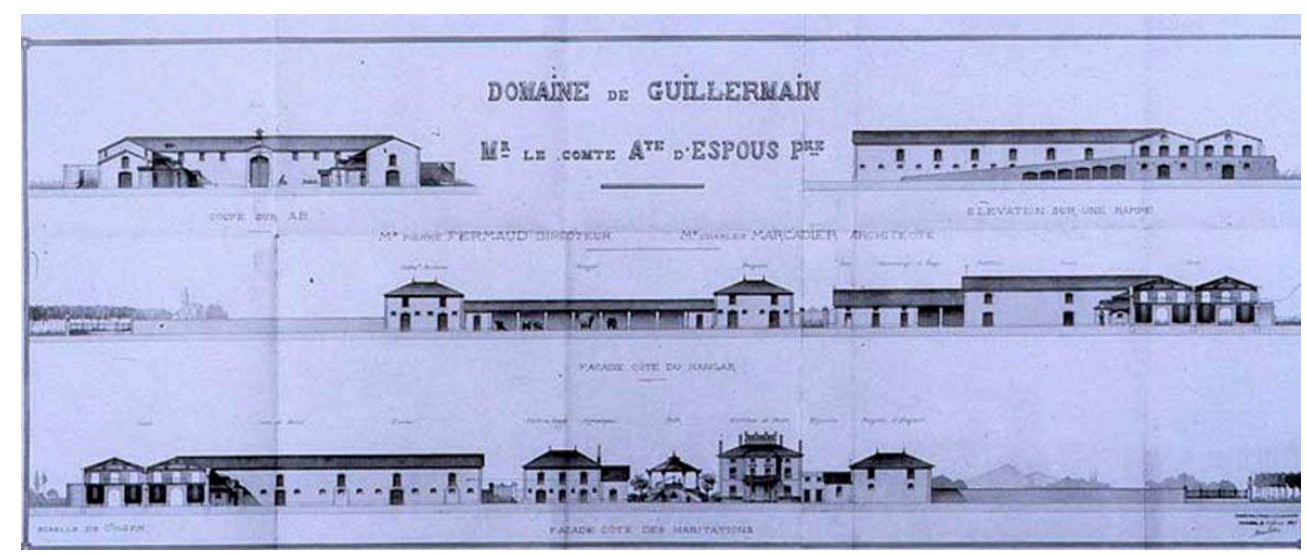

Mas de Guillermain (Mauguio). Elévations

Phot. Inv. J.C. Jacques @ Inventaire général, ADAGP, 1994 
Figure 6

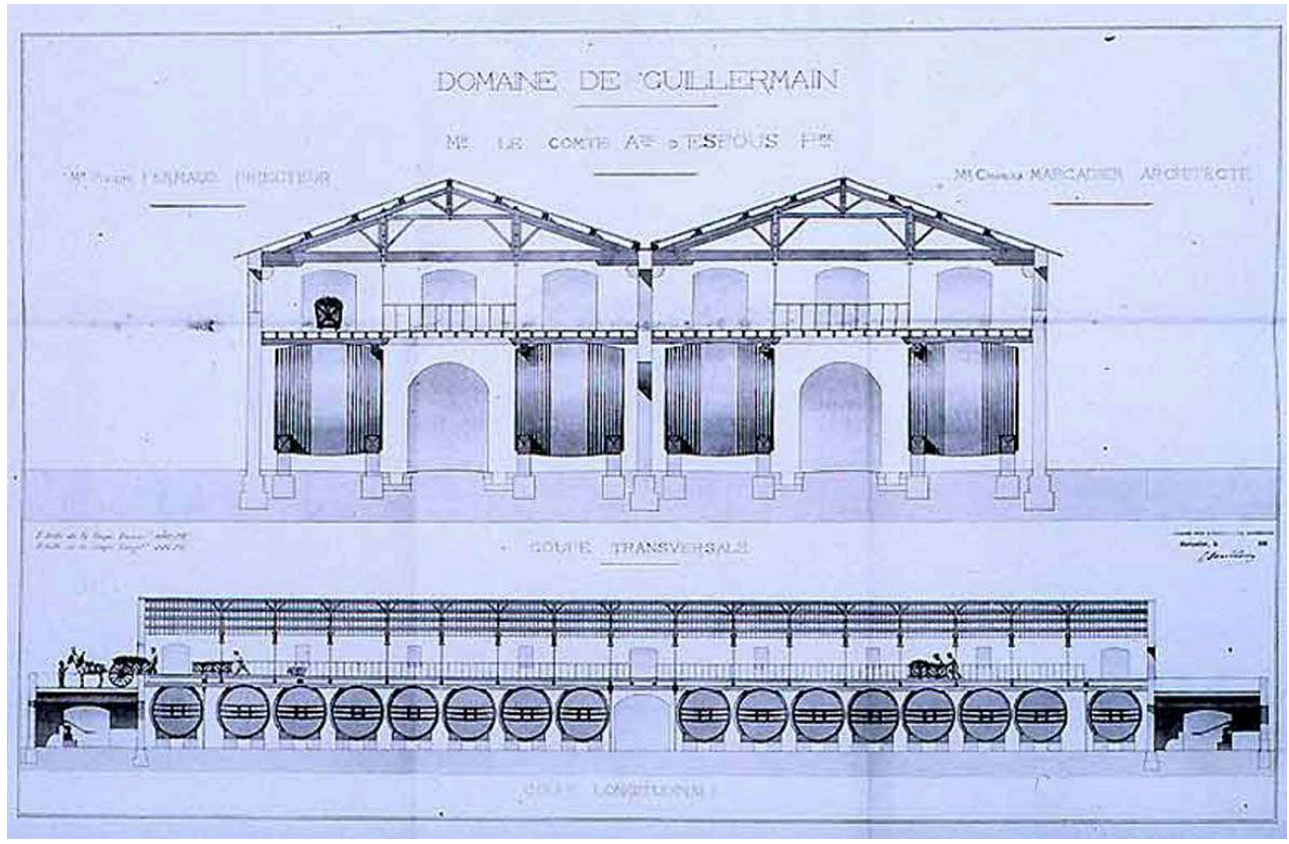

Mas de Guillermain (Mauguio). Coupes transversale et longitudinale sur la cave

Phot. Inv. J.C. Jacques ( ) Inventaire général, ADAGP, 1994

Figure 7

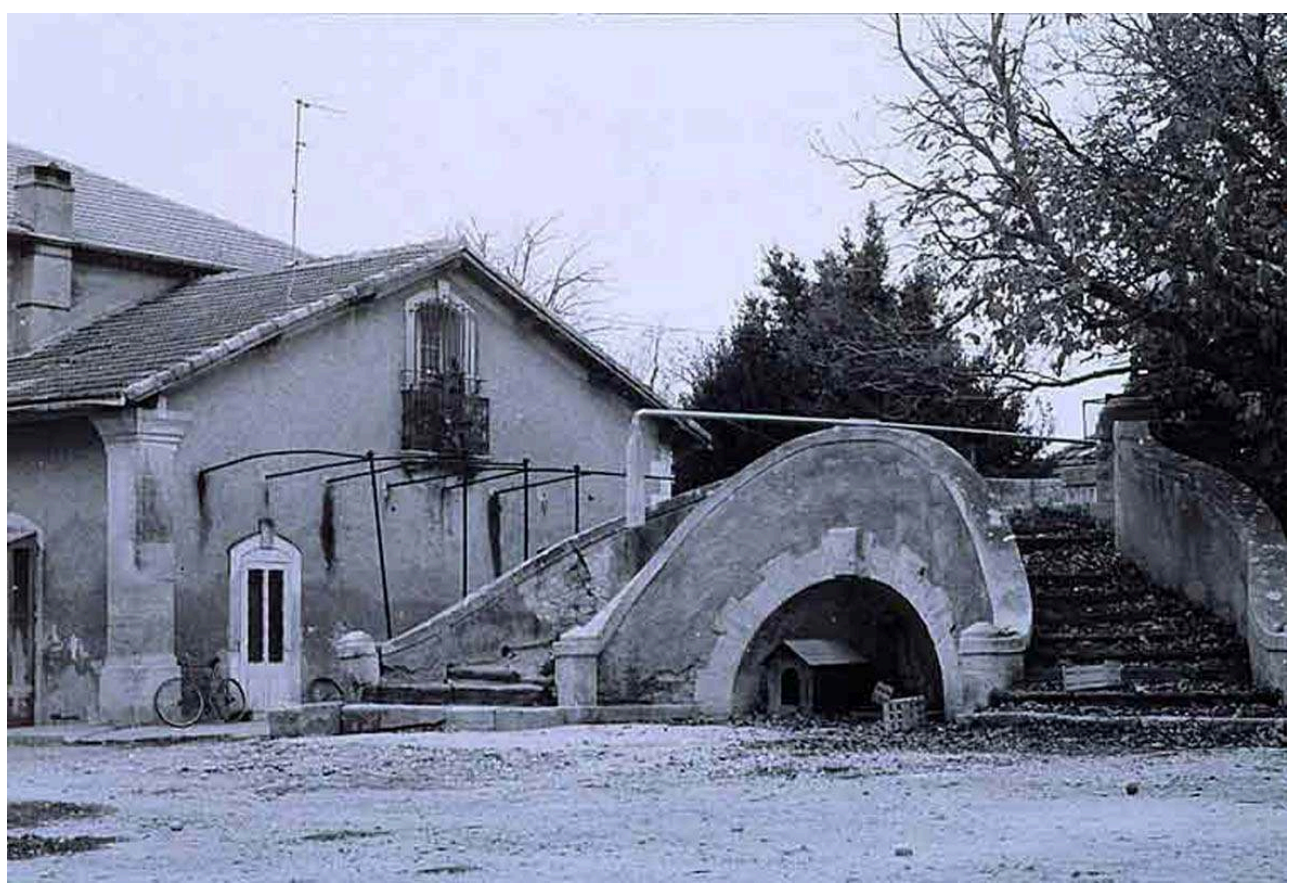

Mas de Guillermain (Mauguio). La noria (photographie prise en 1972)

Phot. Inv. J.C. Jacques @ Inventaire général, ADAGP, 1994

Il serait sans doute intéressant de reprendre ces travaux dans le cadre d'une enquête thématique régionale sur les domaines de la plaine littorale qui permettrait de connaître le nombre et la répartition géographique de ces fermes qui forment de véritables ensembles architecturaux dont une étude historique permettrait de cerner 
tout à la fois la permanence et l'évolution. Aujourd'hui, l'abandon de la monoculture de la vigne et le développement des grandes agglomérations ont entraîné d'importants bouleversements comme la formation de lotissements près des villages et le morcellement de ces immenses bâtiments en habitations individuelles.

Outre les inventaires des cantons d'Aigues-Mortes et de Mauguio, un tel travail pourrait aussi prendre en compte l'étude réalisée par notre service ${ }^{6}$ de quatre cantons audois : Alaigne, Capendu, Limoux et Saint-Hilaire. Cette synthèse était l'aboutissement d'un inventaire thématique enrichi par le dépouillement de sources telles que l'Indicateur des vignobles méridionaux de Charles Gervais, ainsi que par des sondages dans la série $\mathrm{M}$ des Archives départementales (Statistiques agricoles).

\section{La moyenne vallée de l'Hérault : le canton de Gignac ${ }^{7}$}

14 A la suite de l'inventaire topographique du canton de Gignac, les observations générales ${ }^{8}$ qui furent rédigées sur l'habitat des vingt et une communes de ce territoire ont pris en compte la maison paysanne à caractère répétitif des villages et des écarts, la maison bourgeoise ou de notable, et tous les compromis entre ces deux types ainsi que le "mazet" et les fermes du causse d'Aumelas. L'existence de deux thèses importantes sur l'histoire économique et sociale du Languedoc (Dutil, 1911 et Leroy-Ladurie, 1966) a permis de mettre en rapport l'étude de l'habitat avec de solides connaissances, notamment en matière de démographie rurale.

Après avoir analysé les différents types de maisons, ces observations passent en revue tous les caractères architecturaux observés au cours de l'enquête : matériaux et mise en œuvre, combles et couvertures, décor extérieur et intérieur... 
Figure 8

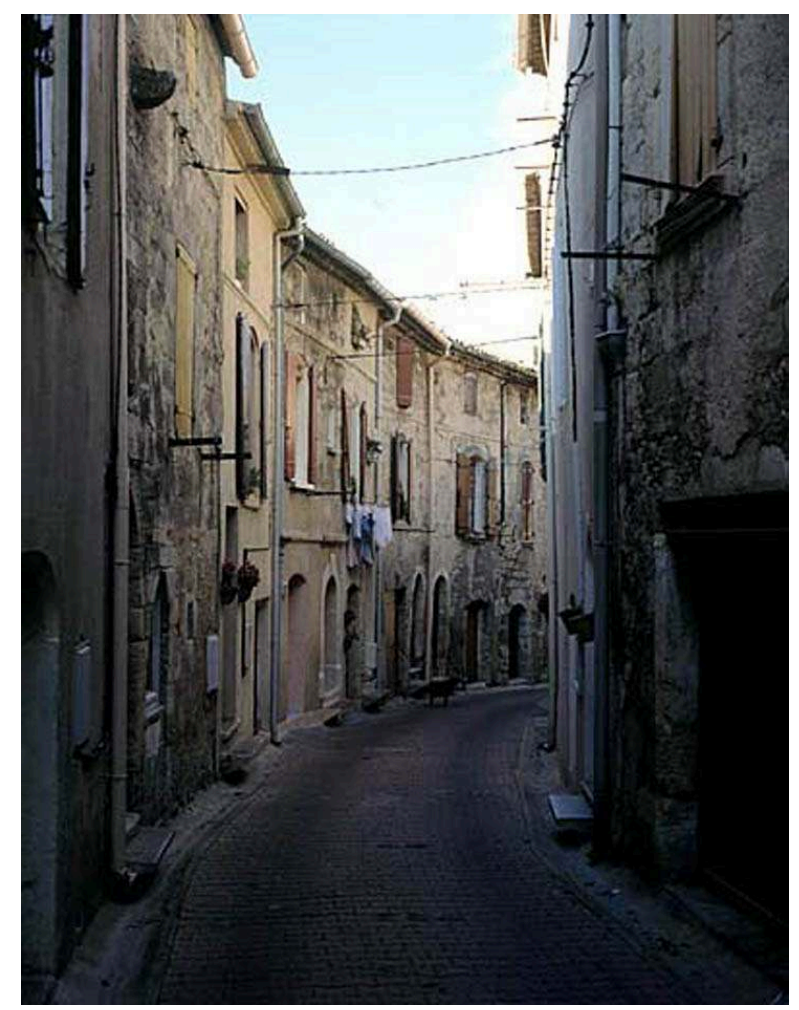

Alignement de maisons élémentaires au Pouget (canton de Gignac)

Phot. Inv. J.M. Périn (C) Inventaire général, ADAGP, 1991

La maison "élémentaire" est située dans les villages où les parcelles sont étroites et allongées, les cours et les espaces libres étant presque inexistants à l'intérieur des enceintes. De petites dimensions (rarement plus de $5 \mathrm{~m}$ de largeur), simple en profondeur, elle ne comporte qu'un seul étage carré posé sur un rez-de-chaussée généralement voûté et surmonté d'un comble en surcroît couvert d'un toit en appentis ; la rareté du bois et l'étroitesse des parcelles ont exclu l'utilisation de fermes. Un escalier droit, très étroit, distribue les trois niveaux à pièce unique, le rez-dechaussée voûté étant réservé aux animaux (mulet ou âne). Des cuves vinaires, dont les parois sont garnies de carreaux de faïence de Saint-Jean-de-Fos occupent parfois une partie de cette " cave ". A l'étage, l'unique pièce d'habitation dispose d'une cheminée en pierre, d'un évier dont l'écoulement se fait directement sur la rue, et d'une alcôve. Le comble abrite les réserves; des coffres pour le grain témoignent de l'existence d'une agriculture disparue. En élévation, ces maisons présentent une disposition caractéristique: porte piétonne butant sur la première volée de l'escalier et porte bâtarde de la cave; au-dessus et dans l'axe, une seule travée de baies. A partir du XVI ${ }^{e}$ siècle, cette maison d'ouvrier agricole ou de petit propriétaire terrien semble avoir colonisé le parcellaire d'origine médiévale à l'intérieur des enceintes villageoises (fig. $\mathbf{n}^{\circ} \mathbf{8}$ ). Elle est toujours couverte d'un enduit à la chaux qui dissimule un appareil grossier fait de moellons calcaires mêlés parfois à des galets et peut prendre la couleur ocre des sables de rivière. L'appareil de pierre de taille est employé plus rarement et semble caractériser les constructions les plus anciennes. Le décor, extrêmement sobre, se limite à des bandes saillantes qui marquent les niveaux et forment les encadrement des baies, souvent en arc segmentaire. Les toits sont couverts de tuile creuse et l'avant- 
toit fermé d'une génoise à deux parfois trois rangs de tuiles creuses. Enfin, dans tout le territoire proche de Saint-Jean-de-Fos, centre actif de production faïencière, on trouvait encore des exemples de chenaux et de gouttières en poterie vernissée de couleur verte.

La tuile creuse, d'usage exclusif dans la plaine et les vallées fluviales, se trouve associée au schiste en couverture dès que l'on aborde les hauteurs du Lodévois et du Larzac. Cette association est probablement le résultat d'une évolution dont l'histoire reste à faire, qui a vu le remplacement progressif de la pierre de couverture par la tuile, beaucoup plus légère (fig. $\mathbf{n}^{\circ} 9, \mathbf{1 0}$ ).

Figure 9

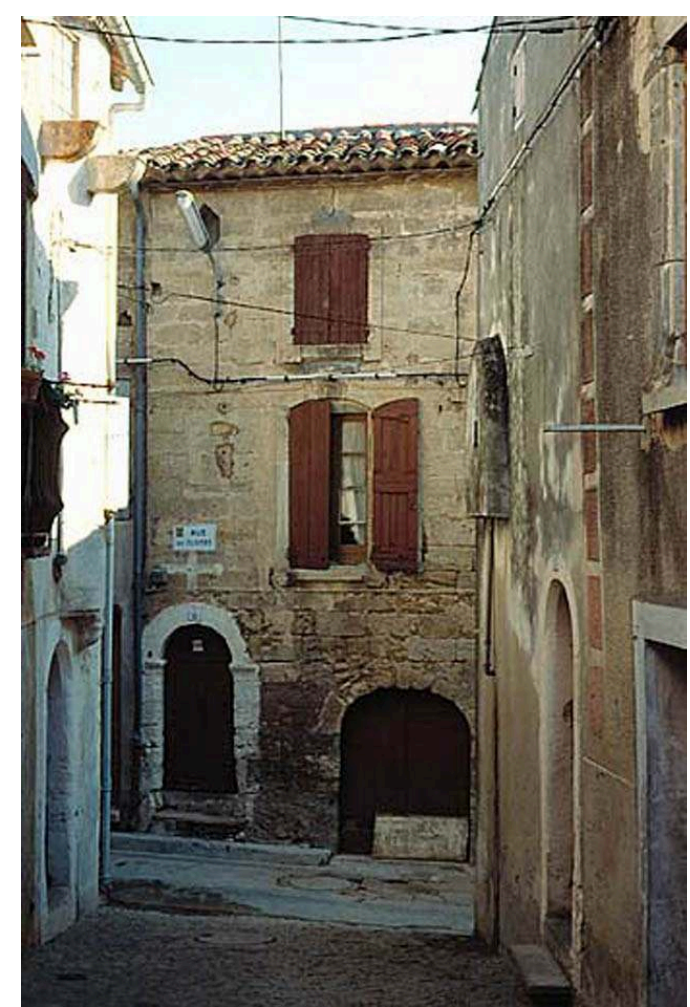

Maison au Pouget (canton de Gignac). Elévation sur rue Phot. Inv. J.M. Périn (c) Inventaire général, ADAGP, 1992 
Figure 10

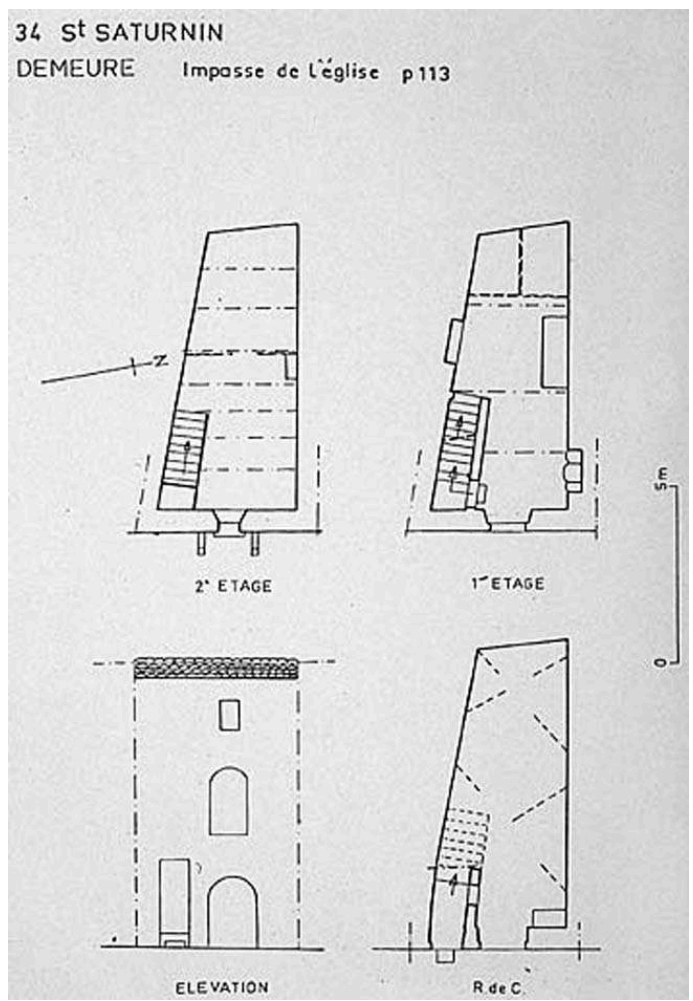

Maison à Saint-Saturnin (canton de Gignac). Plans aux trois niveaux et élévation sur rue, relevé schématique

Phot. Inv. J.C. Jacques @ Inventaire général, ADAGP, 1994

Ces "observations" sur l'architecture du canton de Gignac ont servi de référence aux enquêtes qui ont été faites par la suite en milieu rural : enregistrement des données accumulées sur l'ensemble de l'arrondissement de Lodève et du canton d'Aniane, publication des Images du Patrimoine sur les cantons de Gignac et de Clermont-l'Hérault ${ }^{9}$ et enfin, réalisation de l'inventaire du canton d'Aniane. Elles restent encore aujourd'hui une base de travail pour les enquêtes entreprises sur le patrimoine de la plaine languedocienne et des vallées des fleuves côtiers. Cependant, attachées à dégager et à décrire les différents types de maisons ou de fermes, elles ne donnent qu'un aperçu des villages, reprenant dans le cadre des observations sur les maisons ou sur les fermes des typologies formelles définies depuis longtemps par les géographes: village perché, village de plaine, village-rue. 
Figure 11

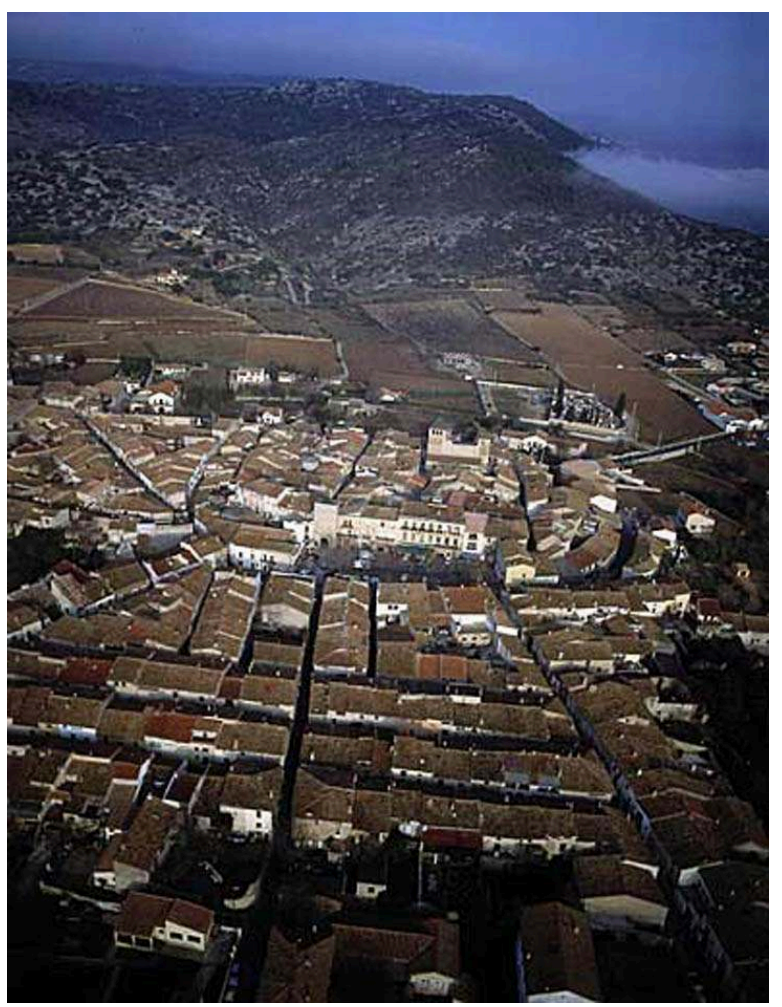

Le village de Saint-Jean-de-Fos. Vue aérienne montrant les extensions successives du village à partir de l'église Saint-Jean et d'une première enceinte de forme grossièrement rectangulaire, datée de 1155 Le faubourg est un lotissement régulier du XIII ou du XIVe siècle. Sur la place publique, située entre l'enceinte et le faubourg, on trouve la porte de l'Horloge, le beffroi communal qui fut restauré en 1747 la mairie construite au XIXe siècle ainsi que la fontaine

Phot. Inv. J.M. Périn (C) Inventaire général, ADAGP, 1991

19 Paradoxalement, le travail sur la ville de Montpellier ${ }^{10}$ a entraîné une réflexion sur la formation des villages qui présentent, dans la plaine languedocienne, une organisation d'ordre urbanistique. Ce sont des ensembles organisés selon des trames complexes d'ordre historique : existence du château ou de l'église à l'origine de l'agglomération, tracé des enceintes, des rues et des routes, qui constituent la trame des villages. L'analyse des plans cadastraux, des vues aériennes (fig. $\mathbf{n}^{\circ} \mathbf{1 1}$ ) et leur mise en rapport avec les documents d'archives permettent de donner une explication aux formes variées et complexes observées et d'ébaucher des typologies. ${ }^{11}$

\section{Le canton du Caylar et le causse du Larzac méridional ${ }^{12}$}




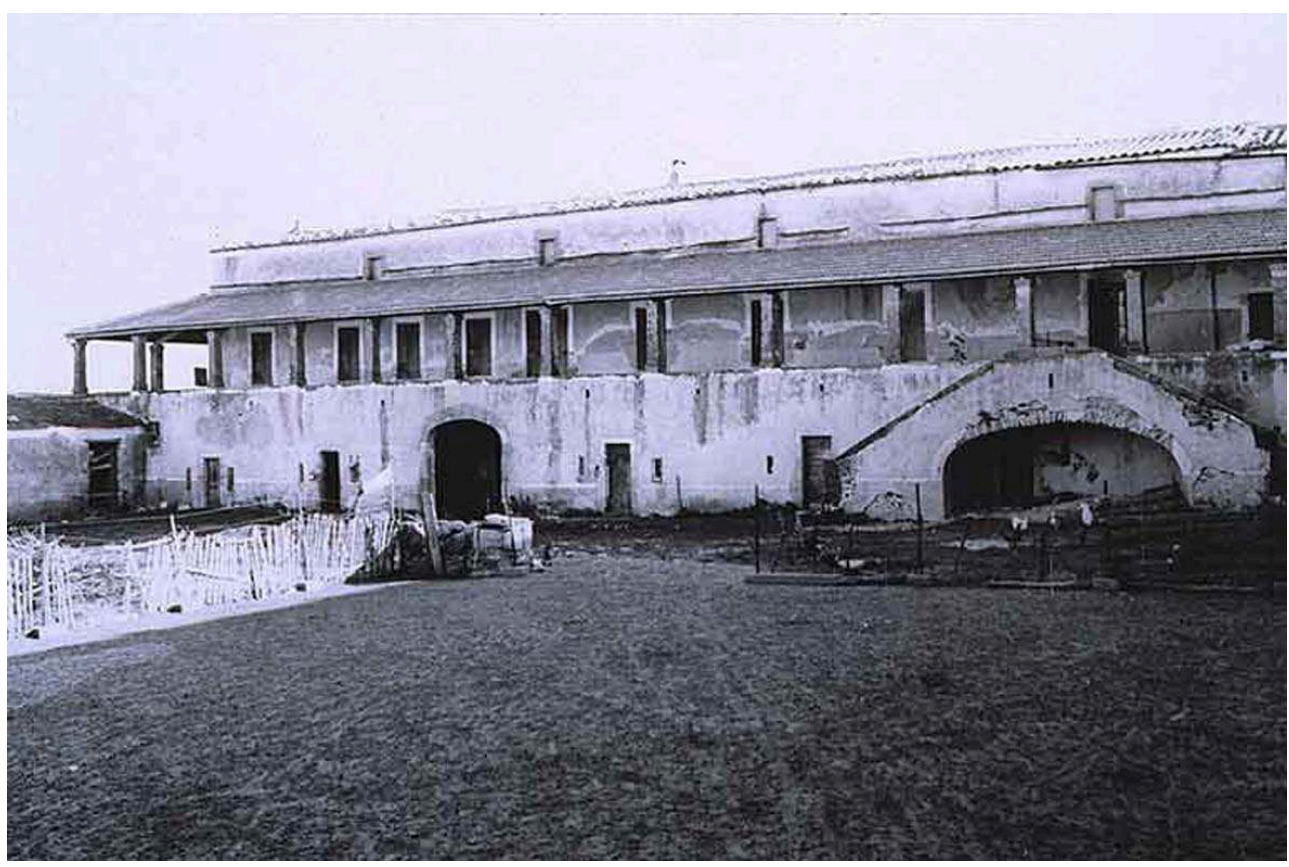

Ferme de La Prade (Saint-Michel d'Alajou). Elévation du logis

Phot. Inv. J.C. Jacques @ Inventaire général, ADAGP, 1994

Dès que l'on quitte la plaine littorale et la vallée de l'Hérault pour le piémont et le plateau du Larzac, le mode d'implantation de l'habitat change. Sur ce vaste plateau karstique au relief mamelonné, les fermes sont groupées en petites agglomérations ou, plus rarement, isolées au milieu de domaines consacrés à l'élevage des moutons et à la transhumance. Cependant, la présence d'un petit nombre d'aires à battre dallées témoigne de la culture aujourd'hui disparue des céréales. Le pré-inventaire de ce canton a permis d'étudier une architecture paysanne caractérisée par l'emploi systématique de la pierre calcaire pour le gros œuvre et par l'usage de la voûte en berceau; les charpentes sont réduites au minimum, les fermes étant remplacées par des arcs diaphragme afin d'économiser le bois dans un pays où la forêt a très tôt disparu. Cette technique permettait de supporter le poids considérable des toits couverts de dalles de calcaire dites lauzes. Ces dernières sont souvent remplacées ou associées à la tuile creuse, plus légère. La ferme de La Prade (commune de Saint-Michel d'Alajou) qui fut sans doute pour partie construite au XVIII siècle (fig. $n^{\circ} 12$ ), est mentionnée sur la carte de Cassini.

Elle était entièrement consacrée à l'élevage ovin. Au moment de l'enquête de 1976, elle servait d'hébergement temporaire aux bergers transhumants et à leurs troupeaux. Adossée à un relief, cette grande bâtisse adopte un plan régulier en U fermé par un mur. Elle est caractérisée par des niveaux superposés de bergeries, et par l'importance du logis situé à l'étage et desservi par une galerie haute (fig. $n^{\circ} 13,14,15$ ). 
Figure 13

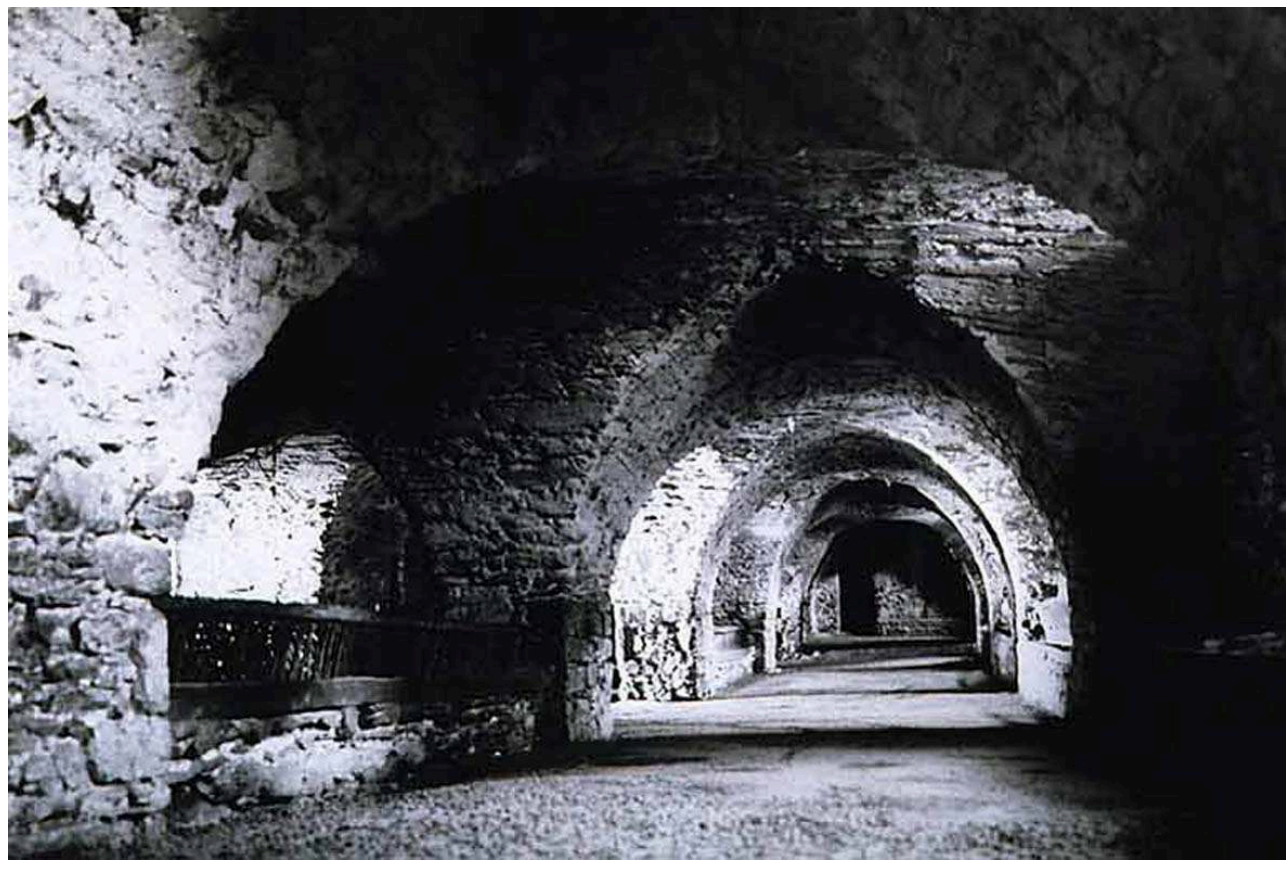

Ferme de La Prade (Saint-Michel d'Alajou). Premier niveau, bergerie voûtée en berceau à pénétrations Phot. Inv. J.C. Jacques (@) Inventaire général, ADAGP, 1994

Figure 14

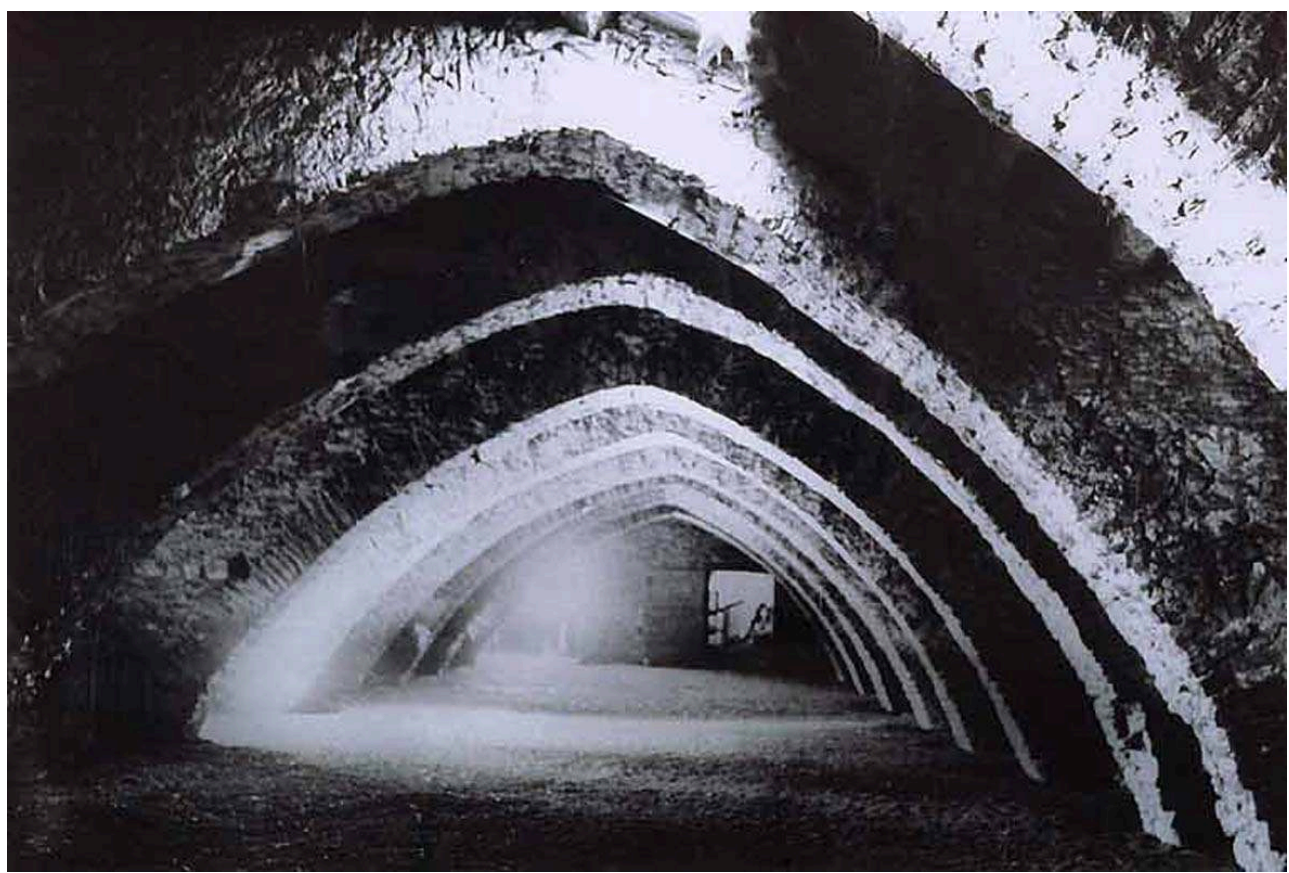

Ferme de La Prade (Saint-Michel d'Alajou). Troisième niveau, bergerie haute dans le comble. Des arcs diaphragmes soutiennent le toit

Phot. Inv. J.C. Jacques @ Inventaire général, ADAGP, 1994 
Figure 15

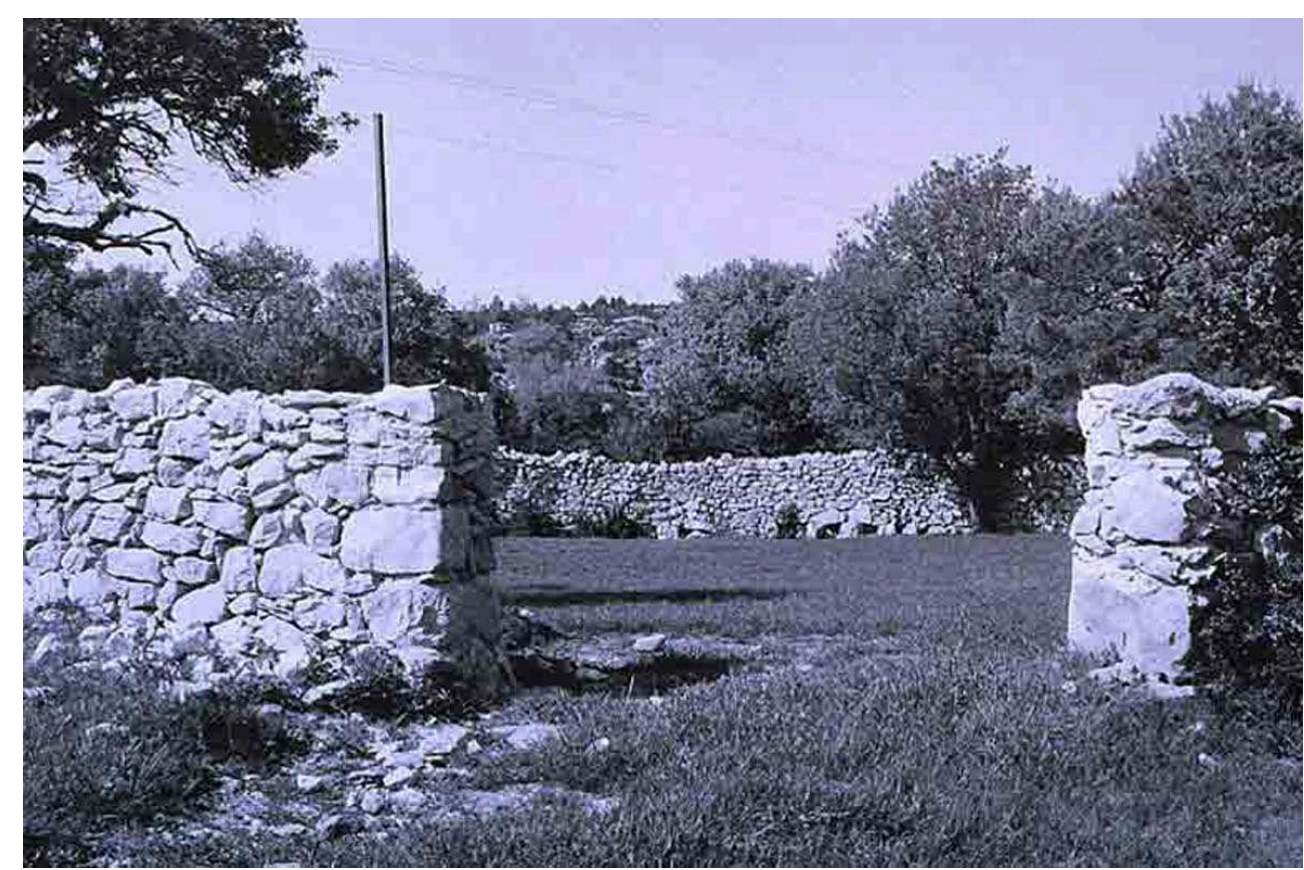

Enclos de pierre sèche sur le causse du Larzac

Phot. Inv. J.C. Jacques @ Inventaire général, ADAGP, 1994

\section{L'inventaire dans le département de la Lozère}

Le territoire du département présente des paysages de moyenne montagne aux caractères fortement individualisés par la géologie. On distingue en effet traditionnellement trois territoires principaux : la Lozère du schiste qui comprend les Cévennes, les zones granitiques du mont Lozère et de la Margeride, enfin les causses calcaires. Les sept cantons inventoriés occupent au centre du département une bande qui s'étend depuis l'Aveyron à l'ouest jusqu'à la limite de l'Ardèche à l'est. Ce territoire est traversé par le Lot et par un affluent de la Loire, l'Altier. Cette "coupe" transversale du département présente un échantillonnage de l'habitat des trois zones géographiques ainsi que de territoires de transition, comme le canton de Villefort ${ }^{13}$ où l'on passe des Cévennes schisteuses dominées par la châtaigneraie aux plateaux granitiques du mont Lozère. 
Figure 16

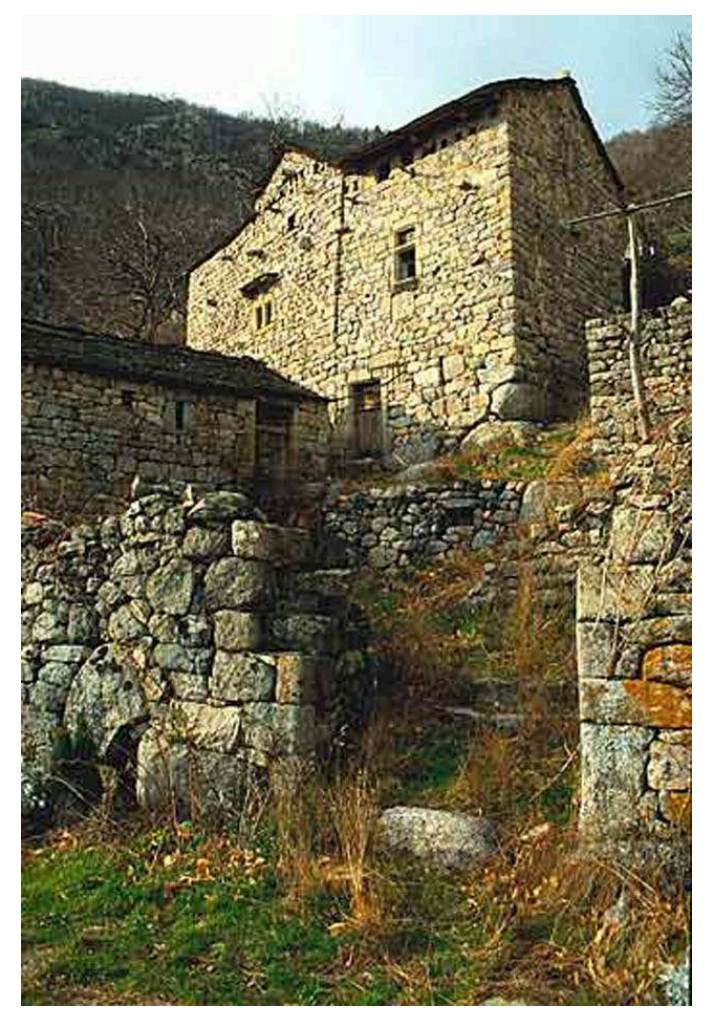

Vue d'ensemble de la ferme de La Viale (Pied-de-Borne)

Phot. Inv. J.M. Périn ( I Inventaire général, ADAGP, 1988

Sur ce dernier territoire, la ferme de La Viale, commune de Pied-de-Borne (fig. $\mathbf{n}^{\circ} \mathbf{1 6}$ ), est un bel exemple d'habitat de granite lié à l'aménagement des terrasses. Elle fut construite au début du XVII e siècle mais un élément en remploi portant la date de 1556 indique la présence d'un premier édifice. La disposition en hauteur du bâtiment principal est adaptée à la pente abrupte de la vallée de la Borne, aménagée en terrasses de culture occupées par la châtaigneraie. Chaque niveau correspond à une terrasse : la cave au premier niveau, surmontée au second par l'unique pièce d'habitation, le troisième niveau étant un comble qui ouvre de plain-pied sur une troisième terrasse et abrite une bergerie. Le mur pignon s'ajoure vers la vallée, la couverture est en lauzes de schiste. Les autres bâtiments de la ferme sont répartis sur les terrasses (deuxième logis, bergerie, séchoir à châtaignes). L'ensemble était fermé par une clôture de pierre sèche dans laquelle une entrée était ménagée au niveau inférieur. Les montants en granite de la porte charretière sont encore en place. 


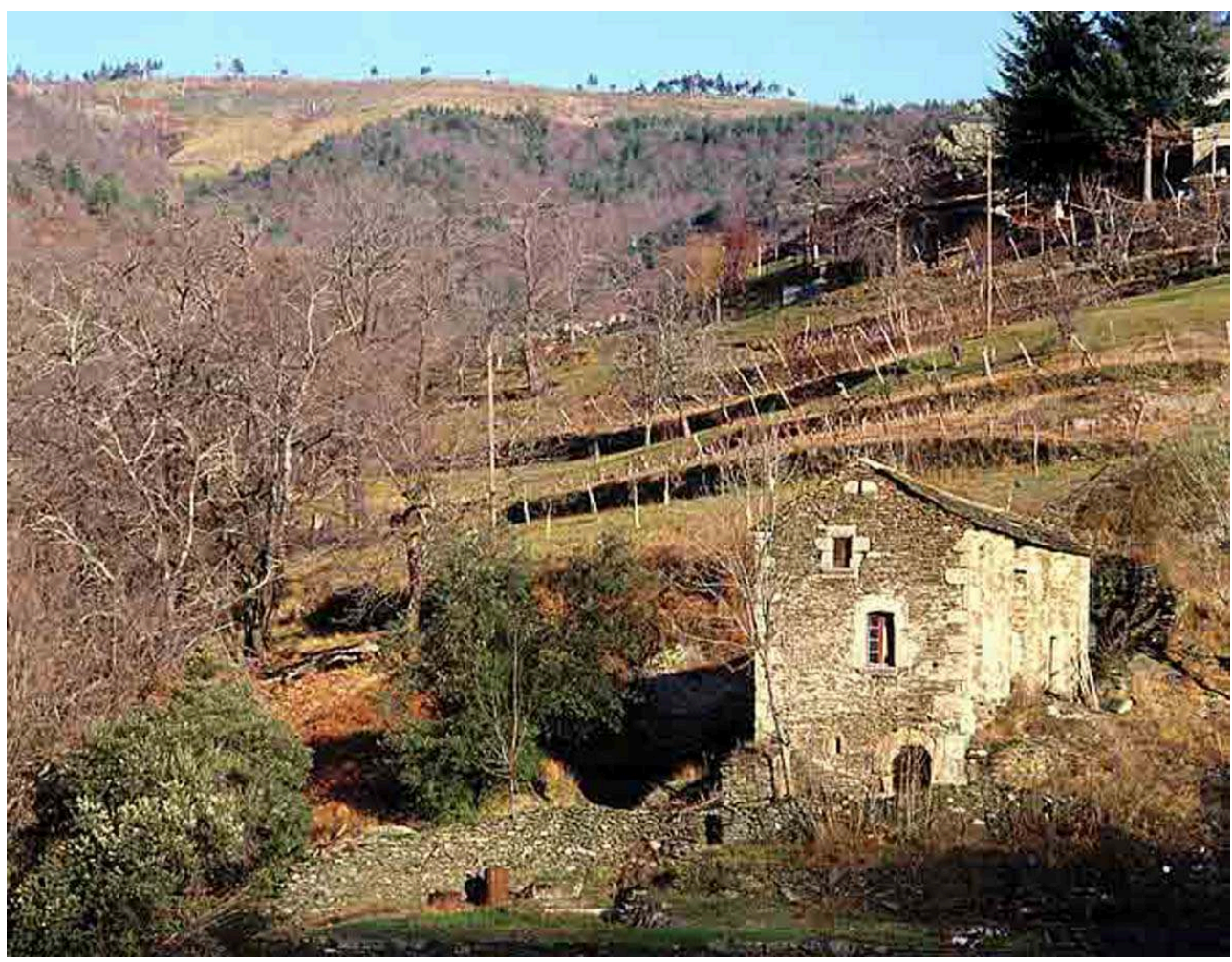

Vue d'ensemble du mas de Jonas (Saint-André-Capcèze)

Phot. Inv. J.M. Périn ( ) Inventaire général, ADAGP, 1988

24 La petite ferme isolée de Jonas (fig. $\mathbf{n}^{\circ}$ 17), sur la commune de Saint-André-Capcèze, est construite selon le même principe au milieu d'un paysage de terrasses exposées au sud où voisinent la vigne et les châtaigniers. Ici l'appareil mêle le schiste et le grès, ce dernier matériau formant les chaînes d'angle et les encadrements des baies.

\section{Le causse de Sauveterre}

Quatre cantons se partagent le causse de Sauveterre et la vallée ${ }^{14}$. Les territoires de la plupart des communes s'étendent à la fois sur la montagne et la vallée aux économies complémentaires. Nous y avons découvert un patrimoine rural de très grande qualité par son ancienneté et par ses caractères architecturaux. La thèse de Paul Marres ${ }^{15}$ sur les Grands Causses, publiée en 1935, reste la meilleure synthèse sur l'histoire et sur la formation de l'habitat de ces territoires. Les sources sont rares : très peu de compoix, les registres des notaires et les archives de l'évêché de Mende restent à dépouiller du point de vue de l'histoire du patrimoine rural.

L'économie y a longtemps été fondée sur l'élevage des moutons pour la laine qui était travaillée dans les fermes et dans les bourgs situés au pied du causse tels que La Canourgue ou Chanac. Cette activité était associée à la culture des céréales dans les dolines et les vallées sèches où s'est accumulée une terre fertile. Les paysans du causse ont pratiqué jusqu'au milieu du XIXe siècle l'assolement triennal (blé ou seigle la première année, orge ou avoine la deuxième, jachère la troisième), qui explique la présence des aires à battre que l'on peut encore repérer en grand nombre. 
27 La culture des plantes fourragères a été introduite à la fin du XVIII ${ }^{\mathrm{e}}$ siècle. Son extension permit le développement à demeure de l'élevage des brebis pour le lait destiné à la fabrication du fromage de Roquefort. Cette transformation a entraîné dans le courant du XIX ${ }^{\mathrm{e}}$ siècle la construction de nouvelles bergeries avec leurs granges et sans doute le remplacement des combles de certains bâtiments anciens voûtés en berceau brisé par de vastes combles charpentés en carène afin d'en augmenter la capacité pour le stockage du fourrage.

On constate la rareté des villages enserrés dans une enceinte urbaine. Cette disposition est réservée aux bourgs, chefs-lieux de canton, de La Canourgue et de Chanac. Les fermes et les maisons sont presque toujours groupées dans un ordre plus ou moins serré à des carrefours de chemins. Ces agglomérations possèdent des espaces communs où se trouvent les fours et les fontaines qui appartiennent encore aux habitants du village. Des domaines ou manses dont la présence est attestée dans l'Acte de paréage de 1307 sont à l'origine de ces écarts que le plan cadastral contemporain appelle villages. Mais l'histoire du développement de ces ensembles est encore lacunaire: les observations de terrain ne permettent pas de donner des datations antérieures au XVII ${ }^{\mathrm{e}}$ siècle. D'après Paul Marres, l'implantation et l'importance des agglomérations sur le causse dépendraient essentiellement de la présence de la terre labourable accumulée au fond des dépressions. Les habitations sont adossées aux pentes rocailleuses des hauteurs dominant les dolines ou les vallées sèches. La création des hameaux, leur importance et l'arrêt de leur développement seraient liés à l'étendue et à la capacité de ces terres mais l'histoire de leur formation reste à faire. Elle est sans doute associée à la création des communautés d'habitants qui sont propriétaires depuis des "temps immémoriaux" de terres (les plus pauvres), d'espaces communs (les coudercs), de fours à pain etc., comme le montre une enquête réalisée en 1686 par l'Intendance du Languedoc. Ce système de propriété perdure, comme dans le nord du département, à travers le système des sectionnaux. Le " couderc" est un espace mal délimité situé le plus souvent à un carrefour. C'est là que l'on trouve les petits édifices tels que le four commun, le travail à ferrer les bœufs et parfois une grande mare ou "lavogne " pour abreuver les bestiaux. Ces derniers sont depuis quelques années l'objet de restauration dans le cadre de mise en valeur du "petit patrimoine". Malheureusement il est très rare que la même attention soit portée aux ensembles. Les fermes qui les composent sont souvent défigurées, par des restaurations abusives, par l'adaptation des bâtiments agricoles aux normes du moment ou par la ruine qu'entraîne leur abandon.

Sur le causse de Sauveterre et sur les petits causses situés au nord du Lot, la rareté du bois et l'abondance de la pierre ont semble-t-il encore une fois entraîné l'utilisation de la voûte aussi bien pour la réalisation des soubassements que pour celle des combles. La structure de base des logis ou des granges-étables est constituée par la superposition de deux voûtes: le rez-de-chaussée est voûté en berceau surbaissé ou voûté d'arêtes, le comble est couvert d'un berceau brisé. Les poussées importantes exercées par cette masse de pierre nécessitent l'érection de murs épais, parfois renforcés par des contreforts (ferme du château de Ressouches, commune de Chanac). La voûte du comble conditionne la pente du toit qui n'est jamais très forte. Dans le corps de logis, le volume supérieur est recoupé par un plancher de façon à créer un espace plafonné destiné à l'habitation (fig. $\left.\mathbf{n}^{\circ} \mathbf{1 8}, \mathbf{1 9}\right)$. 
Figure 18

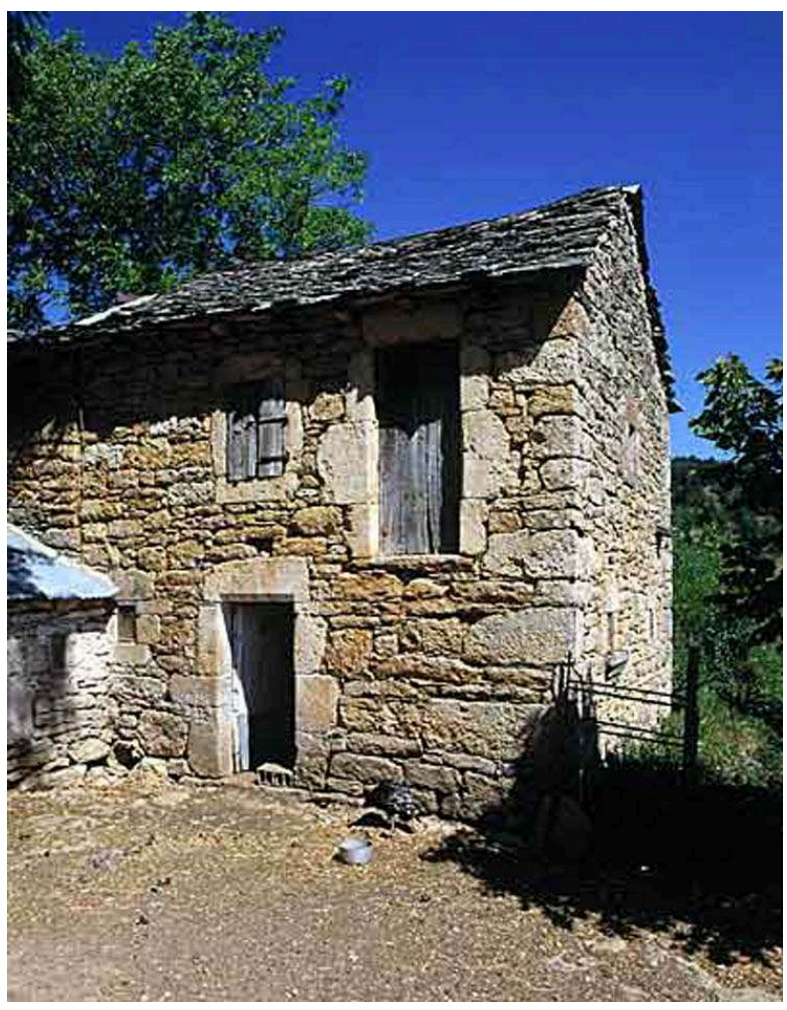

Cultures, hameau du Serre : petite maison d'ouvrier agricole du XVIIe siècle Phot. Inv. J.M. Périn ( ) Inventaire général, ADAGP, 1990 
Figure 19

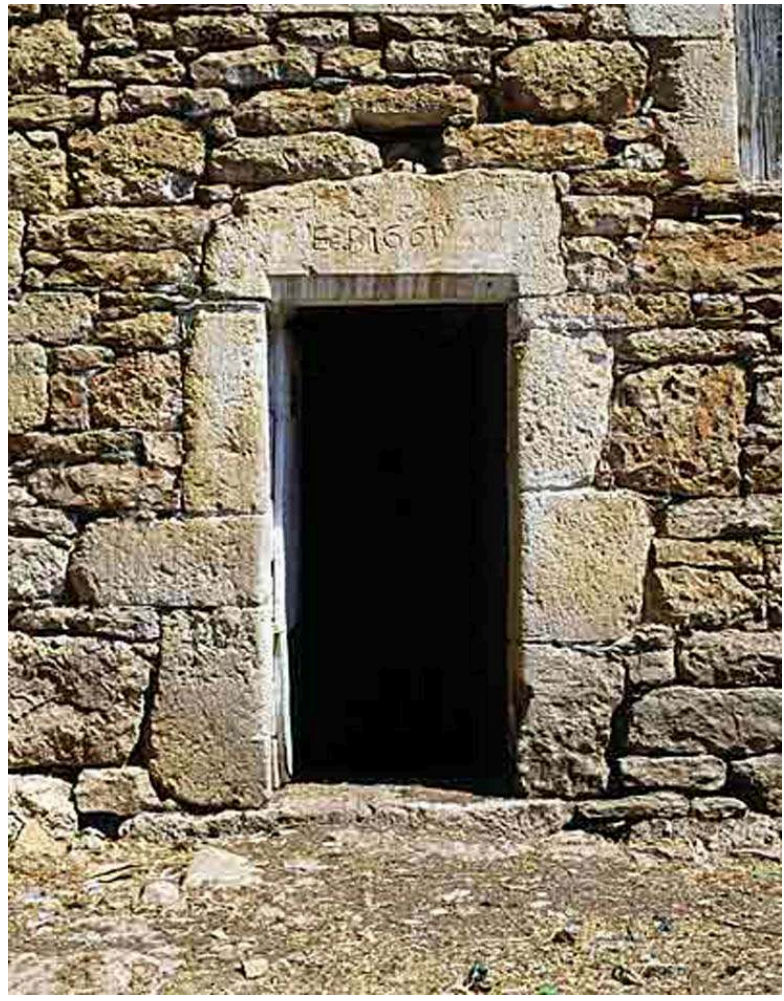

Cultures, hameau du Serre : petite maison d'ouvrier agricole, détail de la porte du logis datée 1661

Phot. Inv. J.M. Périn @ Inventaire général, ADAGP, 1990

30 Trois types de fermes cohabitent dans les villages. Ils correspondent au statut social de leur propriétaire. Les fermes de petits notables, véritables petits manoirs, sont presque partout présentes ; elles voisinent avec les fermes des propriétaires plus modestes et les maisons de journaliers (fig. $\mathbf{n}^{\circ} \mathbf{2 0}$ ).

31 Ces types se différencient par le nombre des corps de bâtiment, par le degré d'ouverture de la cour et par le degré de dépendance du logis par rapport à la grangeétable. Ce dernier se trouve toujours à l'étage d'un corps de bâtiment en hauteur: suivant l'importance de la ferme, l'escalier extérieur débouche sur une terrasse, sur un porche surélevé abritant l'entrée de la cuisine ou sur une galerie haute voûtée (fig. $\left.n^{\circ} 21,22\right)$. 
Figure 20

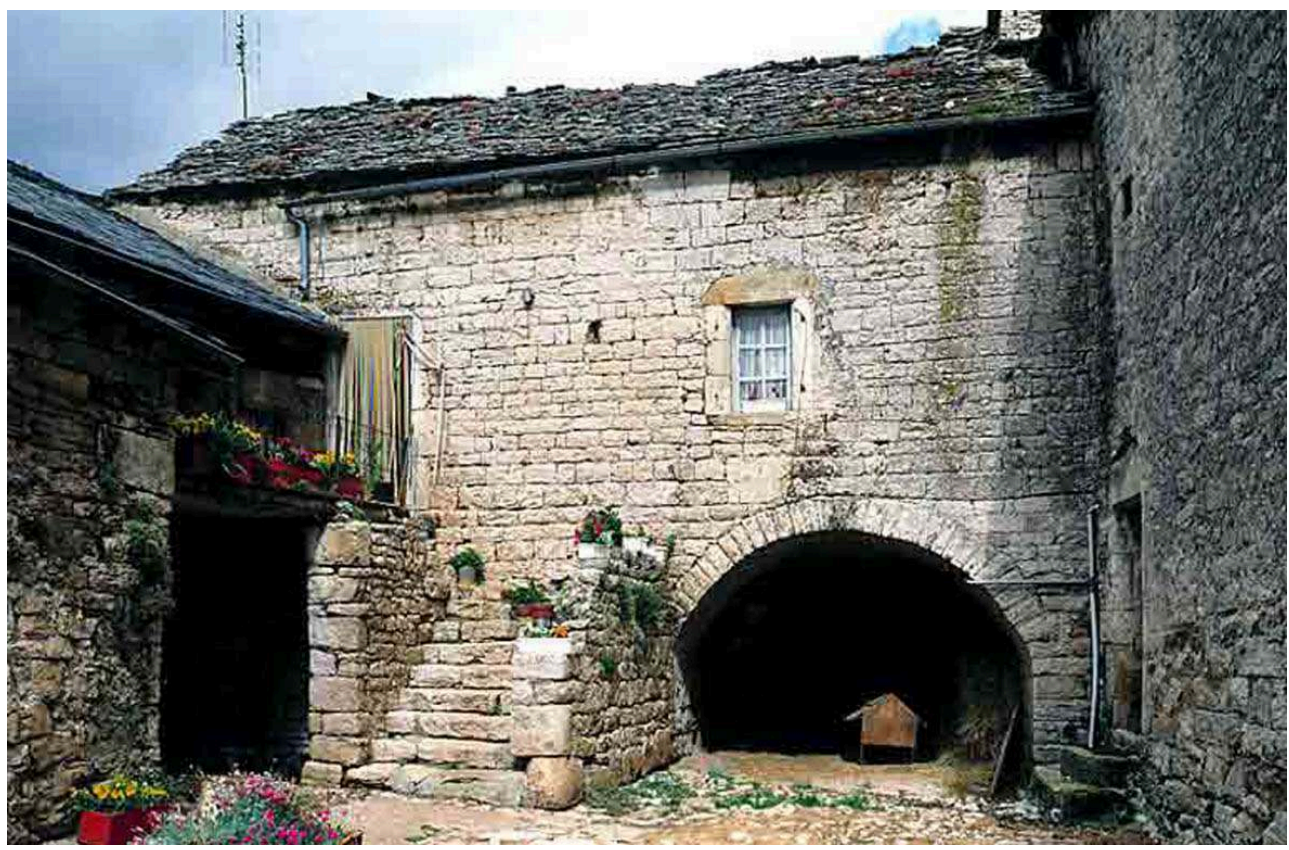

Le Lieuran (Chanac). Ferme datant sans doute du XVIle siècle. La cour est entourée par quatre corps de bâtiment jointifs. On y accède par deux passages couverts voûtés

Phot. Inv. J.M. Périn (c) Inventaire général, ADAGP, 1990

\section{Figure 21}

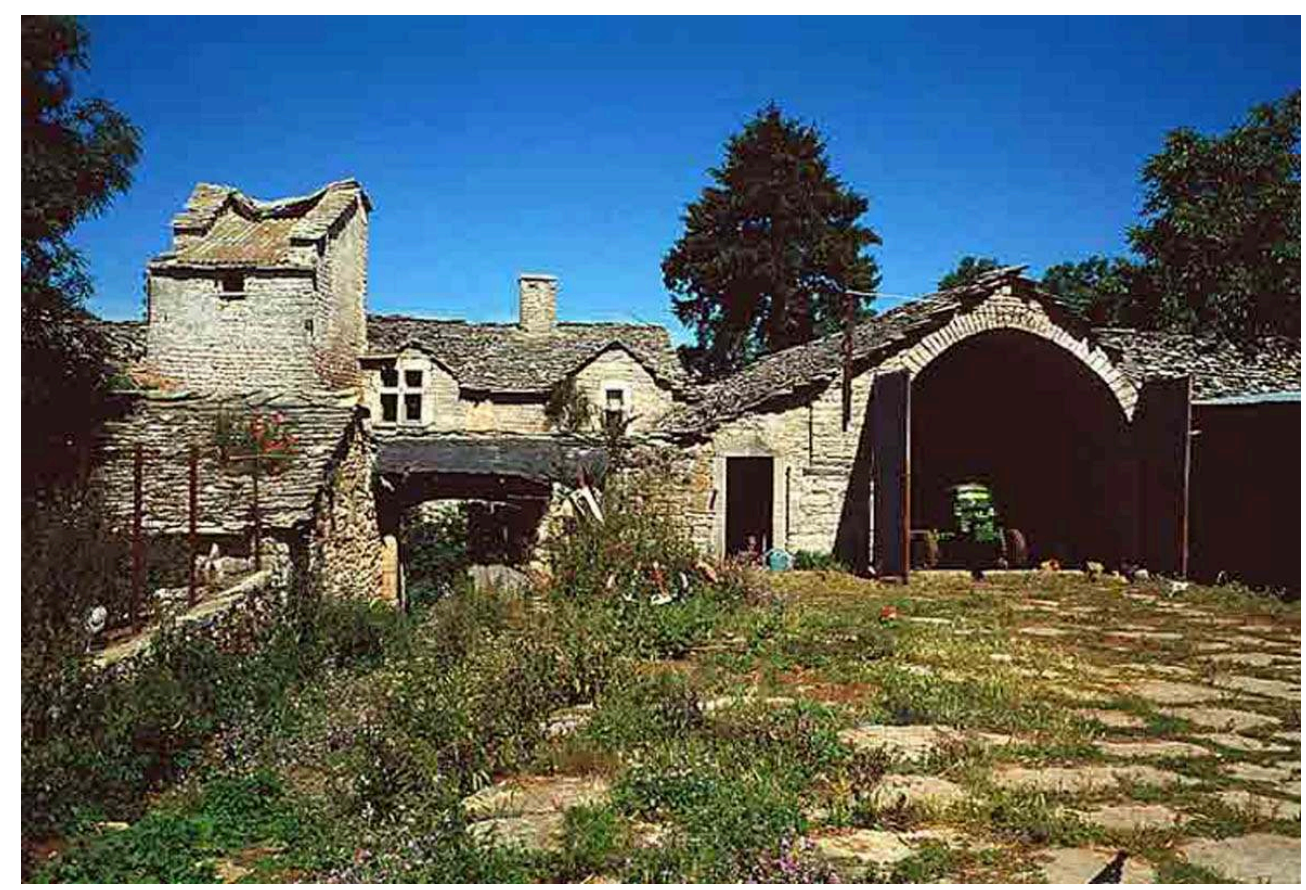

Le Jas (Chanac). Ferme à cour fermée dont l'accès se fait par une porte charretière sous chaperon. Sur le côté, la grange voûtée ouvre sur l'aire à battre. La date de 1650, inscrite sur un linteau de porte à l'intérieur du logis, date probablement l'ensemble de l'édifice

Phot. Inv. J.M. Périn @ Inventaire général, ADAGP, 1990 


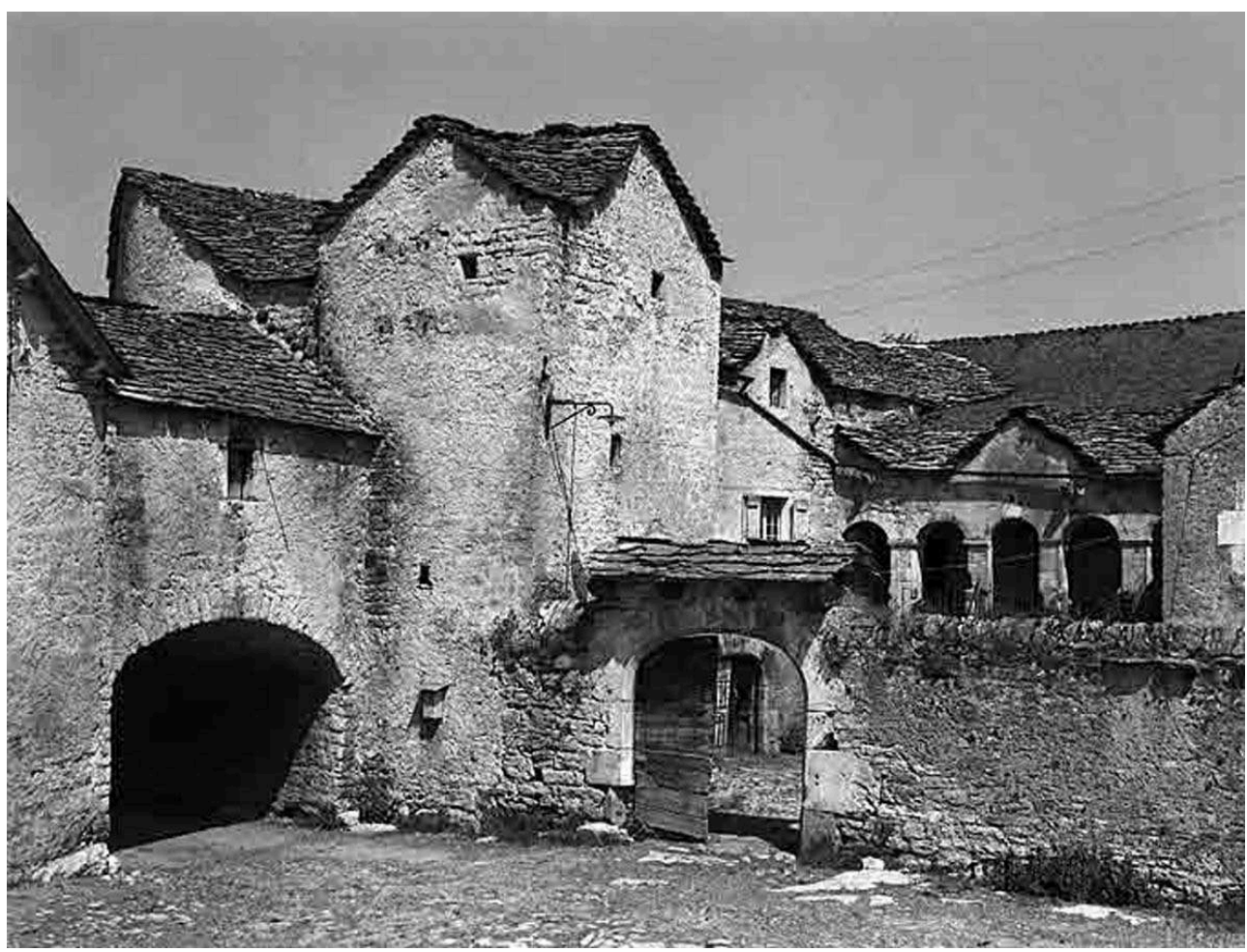

Les Monziols (Saint-Georges de Lévejac). Ferme à galerie haute datant du premier quart du XVIIIe siècle. La photographie prise en 1968 montre un ensemble encore intact. En 1994, le pigeonnier et le passage couvert avaient disparu

Phot. Inv. M. Descossy @ Inventaire général, ADAGP, 1968

\section{La Margeride}

L'inventaire topographique des cantons du Malzieu-Ville et de Saint-Alban-surLimagnole a été réalisé de 1999 à 2002. Leurs territoires forment un cadre géographique et historique d'une grande unité qui ne présente pas de solution de continuité avec les départements voisins du Cantal et de la Haute-Loire (région Auvergne). Il appartient entièrement au massif cristallin de la Margeride, haut plateau vallonné fait de collines occupées par les reboisements du XIX ${ }^{\mathrm{e}}$ siècle, alternant avec des bassins couverts de pâtures et quadrillés par des clôtures de pierre sèche. A l'ouest, la Truyère, dont le cours s'enfonce dans des gorges à partir du Malzieu, borne en partie le territoire. La rivière et les ruisseaux qui l'alimentent actionnaient de nombreux moulins. A l'est, la limite cantonale suit la crête de Margeride qui culmine à plus de 1400 mètres. 
Figure 23

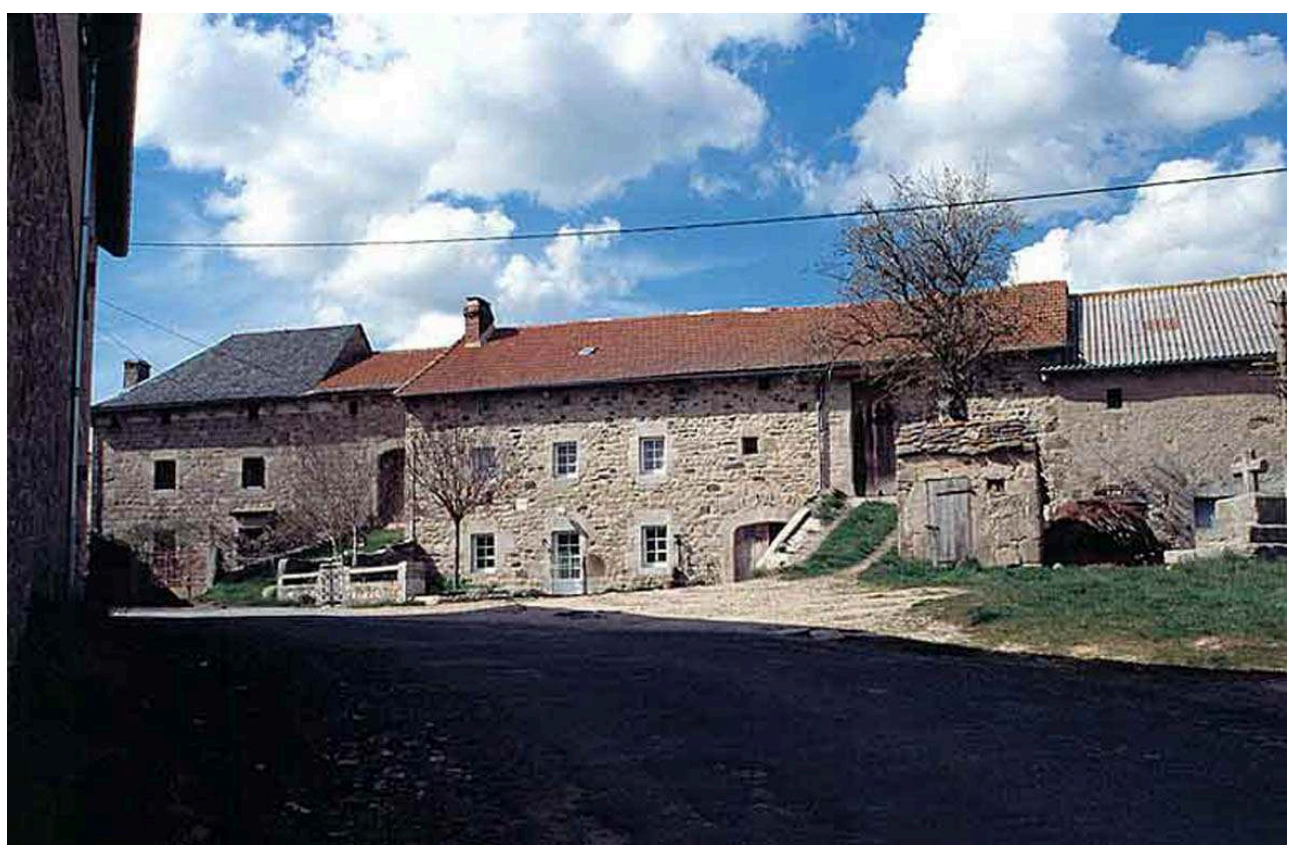

Chaulhac. Alignement de petites maisons jointives appelé " barriade"

Phot. Inv. M.S. Grandjouan (c) Inventaire général, ADAGP, 2000

33 L'agriculture est aujourd'hui dominée par l'élevage des bovins qui a supplanté le système agro-pastoral fondé sur la culture du seigle et l'élevage des ovins. Le patrimoine bâti s'est constitué au cours du XIX ${ }^{e}$ siècle à partir de sites anciens. Comme sur le causse, les fermes sont presque partout rassemblées en petites agglomérations (fig. $\mathbf{n}^{\circ}$ 23).

Les fours à pain, les travails à ferrer, des enclos communs sont les témoins de cette organisation en communautés villageoises (fig. $n^{\circ} 24,25,26$ ). 
Figure 24

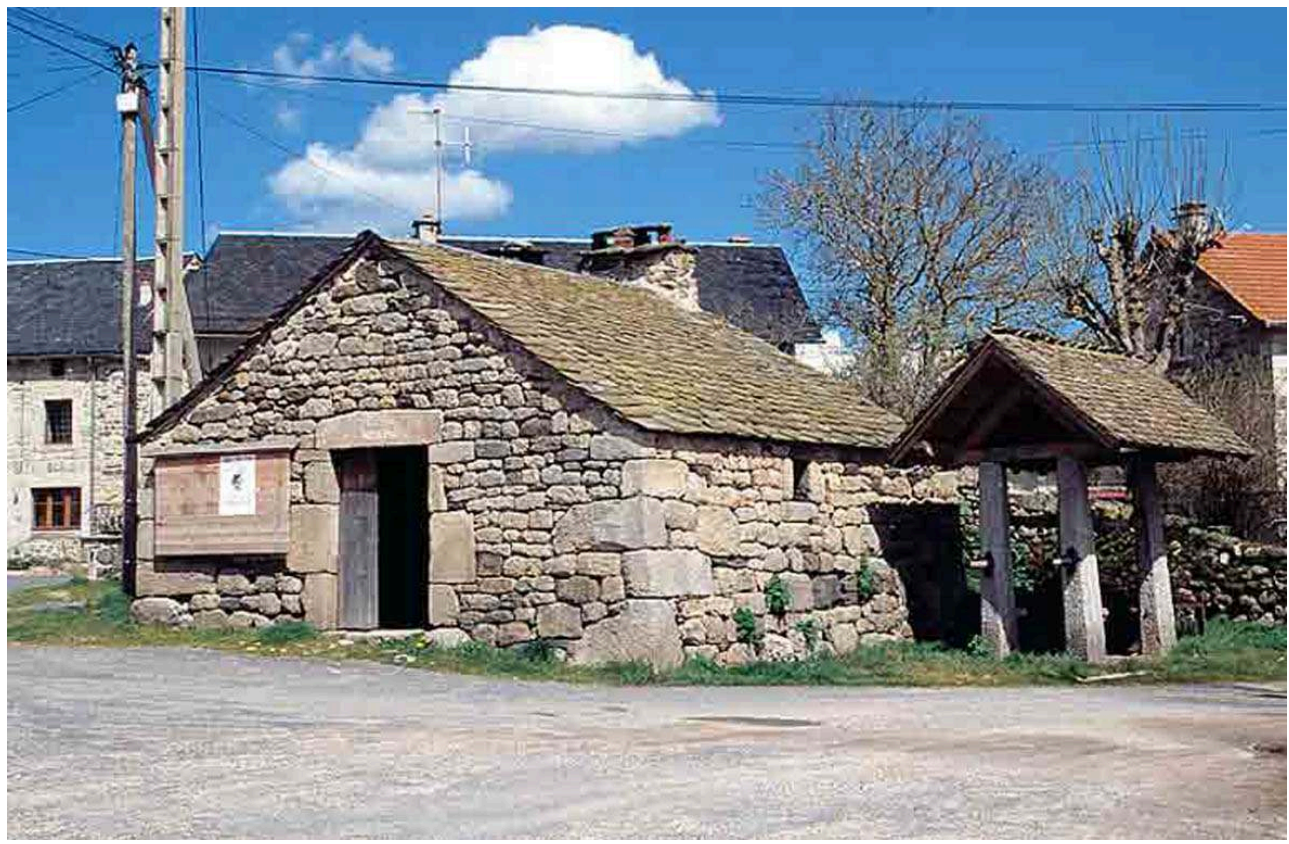

Chaulhac. Espace commun avec four à pain et travail à ferrer

Phot. Inv. M.S. Grandjouan @ Inventaire général, ADAGP, 2000

Figure 25

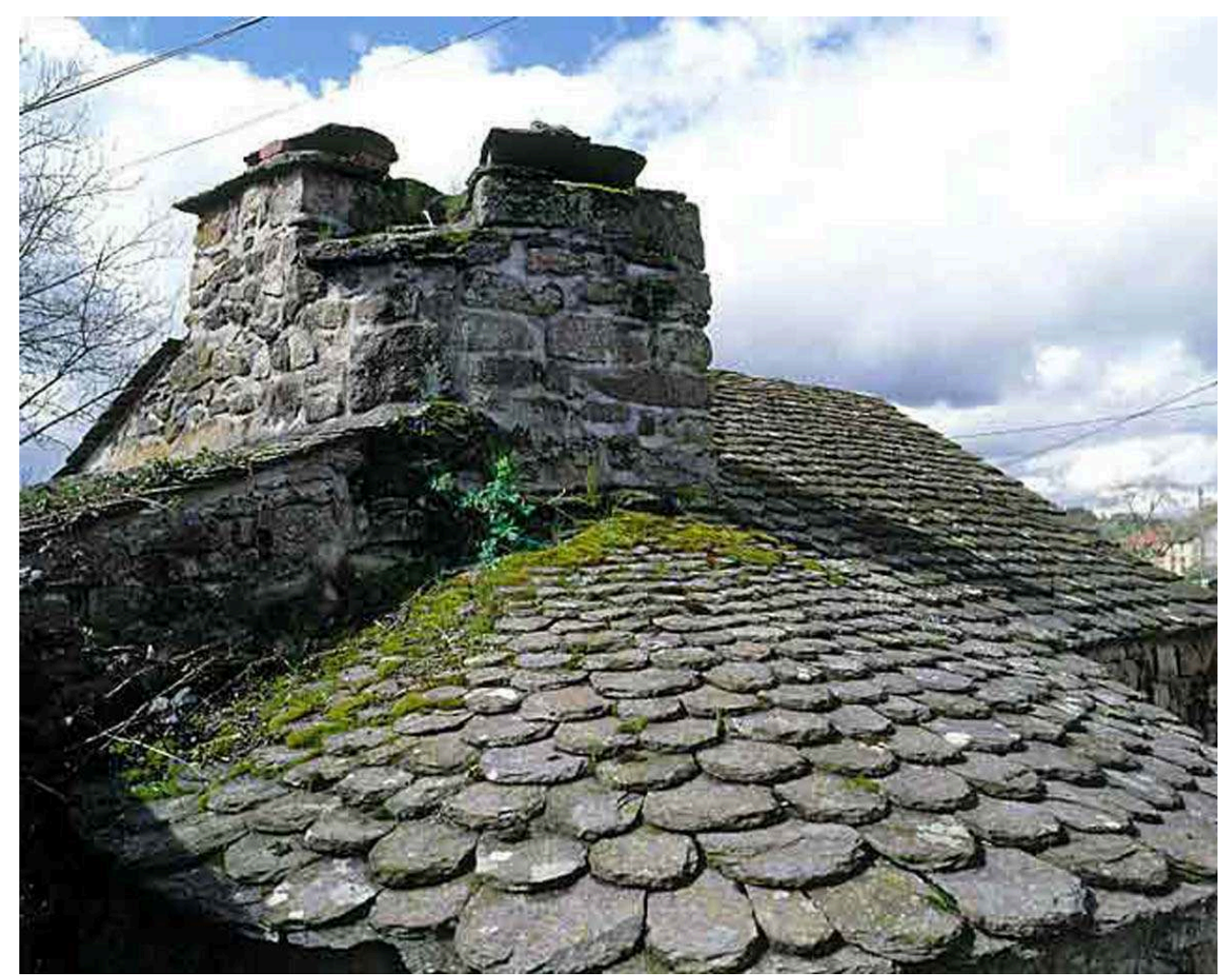

Chaulhac. Le four à pain à deux foyers, détail du toit et des deux cheminées

Phot. Inv. J.M. Périn ( Inventaire général, ADAGP, 2001 
Figure 26

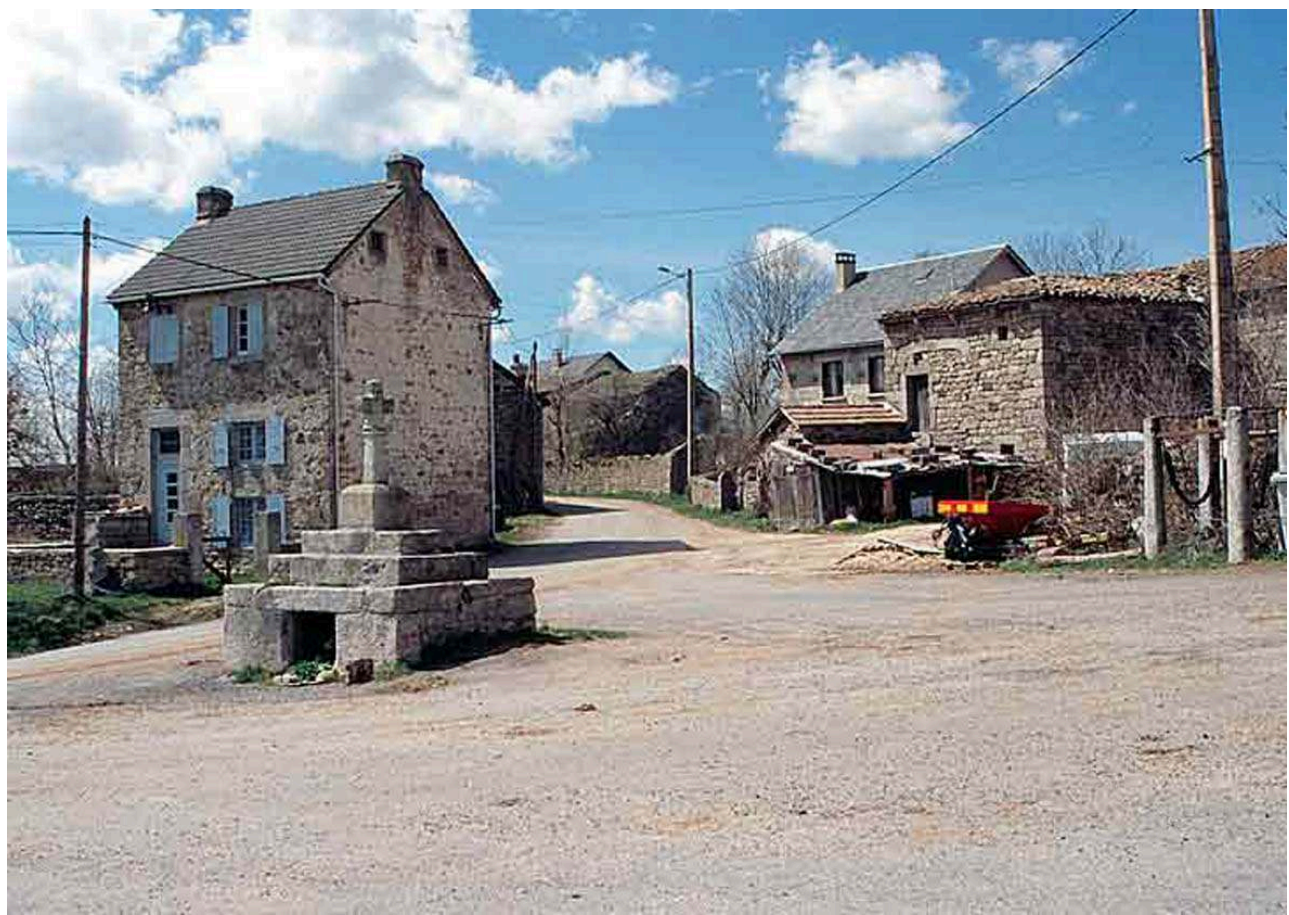

Nozerolles (Chaulhac). Espace commun où sont rassemblés l'école, une croix de chemin et le travail à ferrer

Phot. Inv. M.S. Grandjouan @ Inventaire général, ADAGP, 2000

Dans ces villages, les petites maisons-blocs jointives et alignées en "barres" voisinent avec des fermes plus importantes. La grange et l'étable sont superposées. Les niveaux inférieurs des logis et les étables sont le plus souvent des étages de soubassement creusés dans le sol granitique (fig. $\mathbf{n}^{\circ} \mathbf{2 7}, \mathbf{2 8}$ ). 
Figure 27

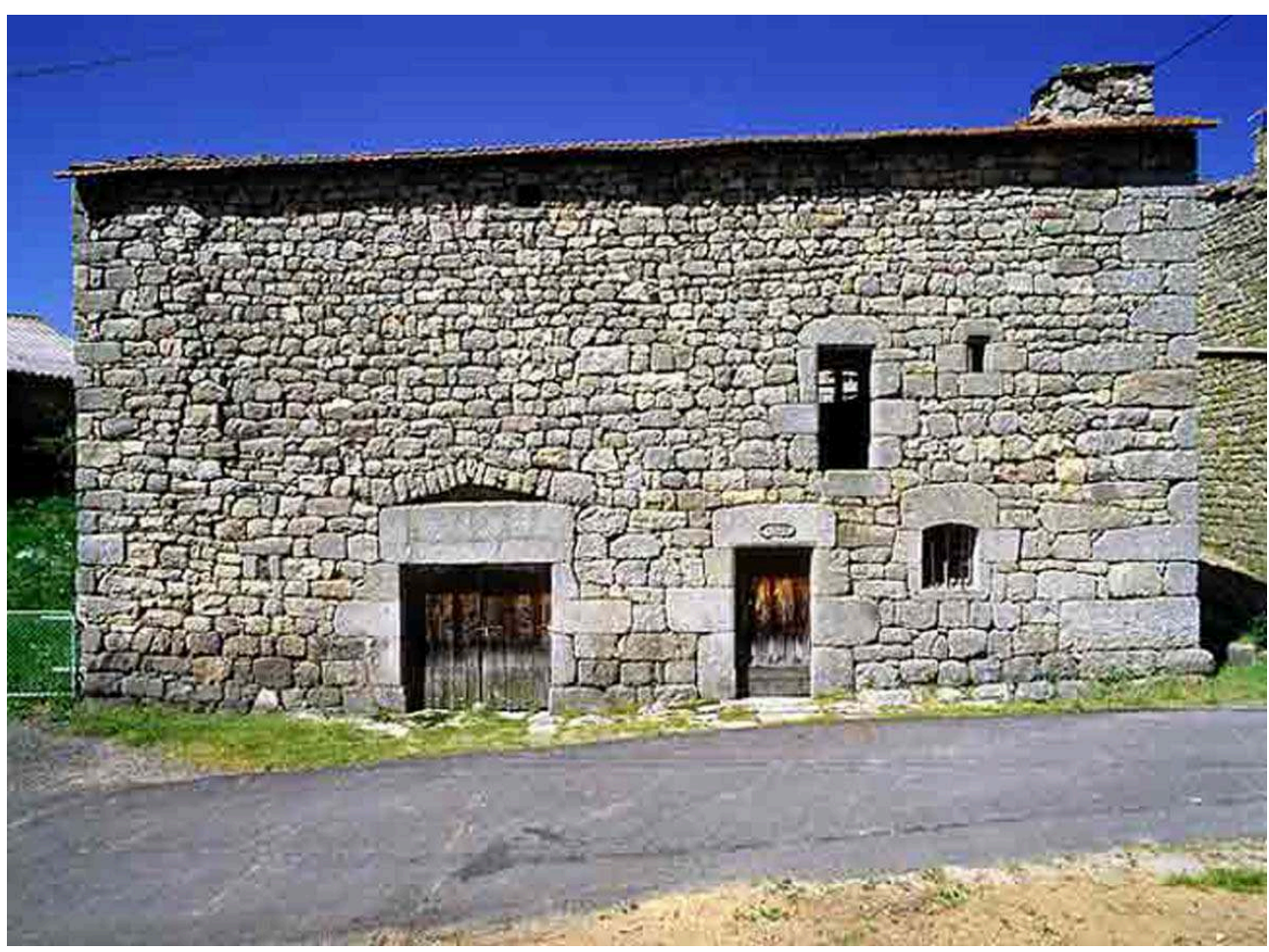

Saint-Privat-du-Fau. Maison datée 1802. Elle comprend un seul bâtiment : logis et étable se partagent l'étage de soubassement, la grange occupe la totalité du rez-de chaussée surélevé. L'entrée principale de la grange se trouve à l'arrière

Phot. Inv. J.M. Périn @ Inventaire général, ADAGP, 2001 
Figure 28

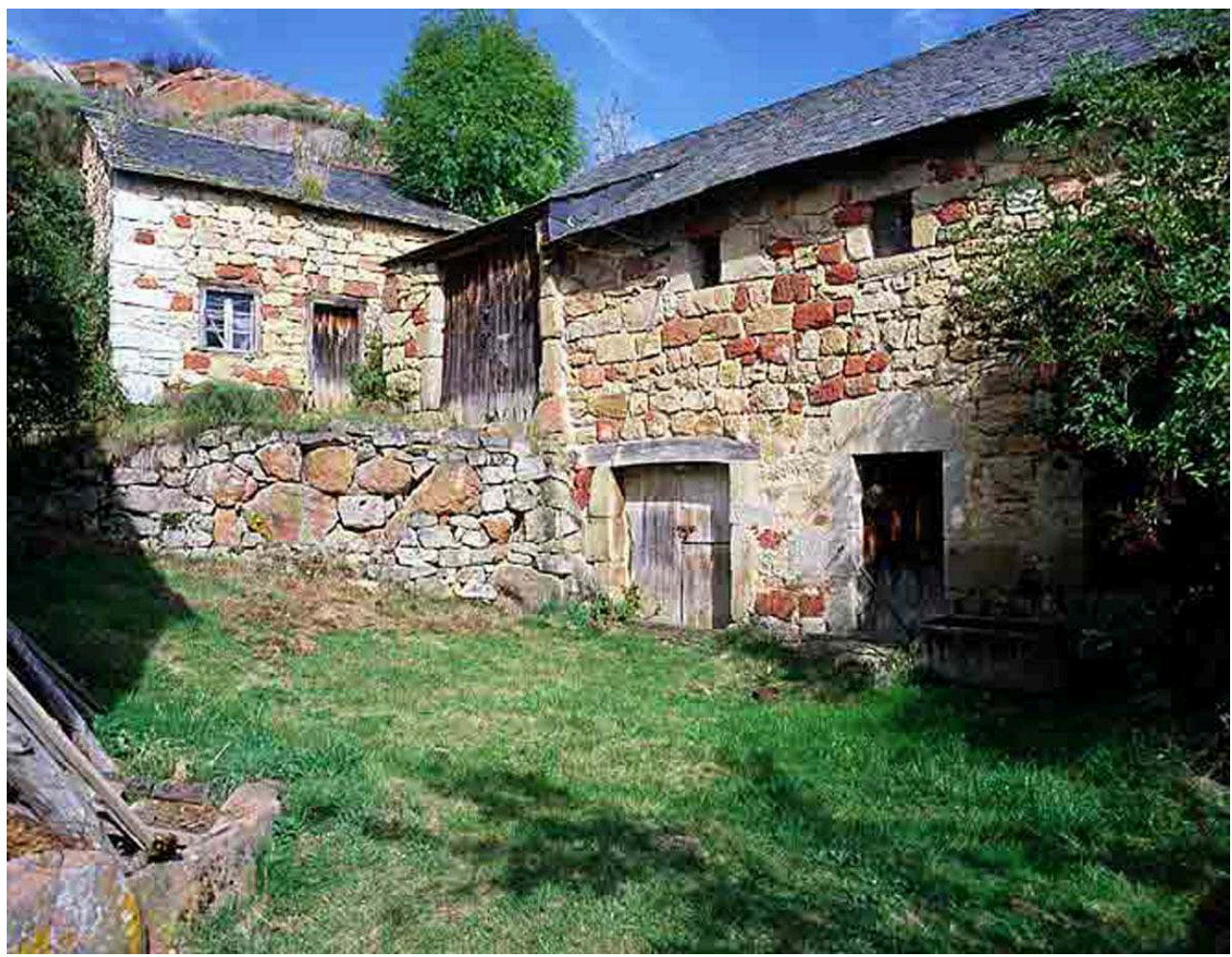

Le Rouget (Saint-Alban-sur-Limagnole). Ferme à bâtiment unique, comme à Saint-Privat. Ici l'accès à la grange se fait en façade par l'intermédiaire d'une rampe. L'appareil de moellons mêle le granite et la " pierre de Rouget ", sorte de grès provenant d'une carrière proche qui a servi pour les embellissements du château de Saint-Alban

Phot. Inv. J.M. Périn (c) Inventaire général, ADAGP, 2001

Le gros œuvre est partout constitué de granite à l'exception de quelques villages au nord du Malzieu où le basalte contraste avec le granite clair des encadrements et des chaînages. Les toits, lorsqu'ils ont conservé leur couverture d'origine, sont couverts de lauzes de schiste ou de chaume de seigle. Autour du Malzieu-Ville, et surtout au sud sur les communes de Serverette et de Saint-Alban, on trouve de grandes fermes isolées au centre d'importants domaines agricoles ${ }^{16}\left(\right.$ fig. $\left.\mathbf{n}^{\circ} \mathbf{2 9}, \mathbf{3 0}\right)$. 
Figure 29

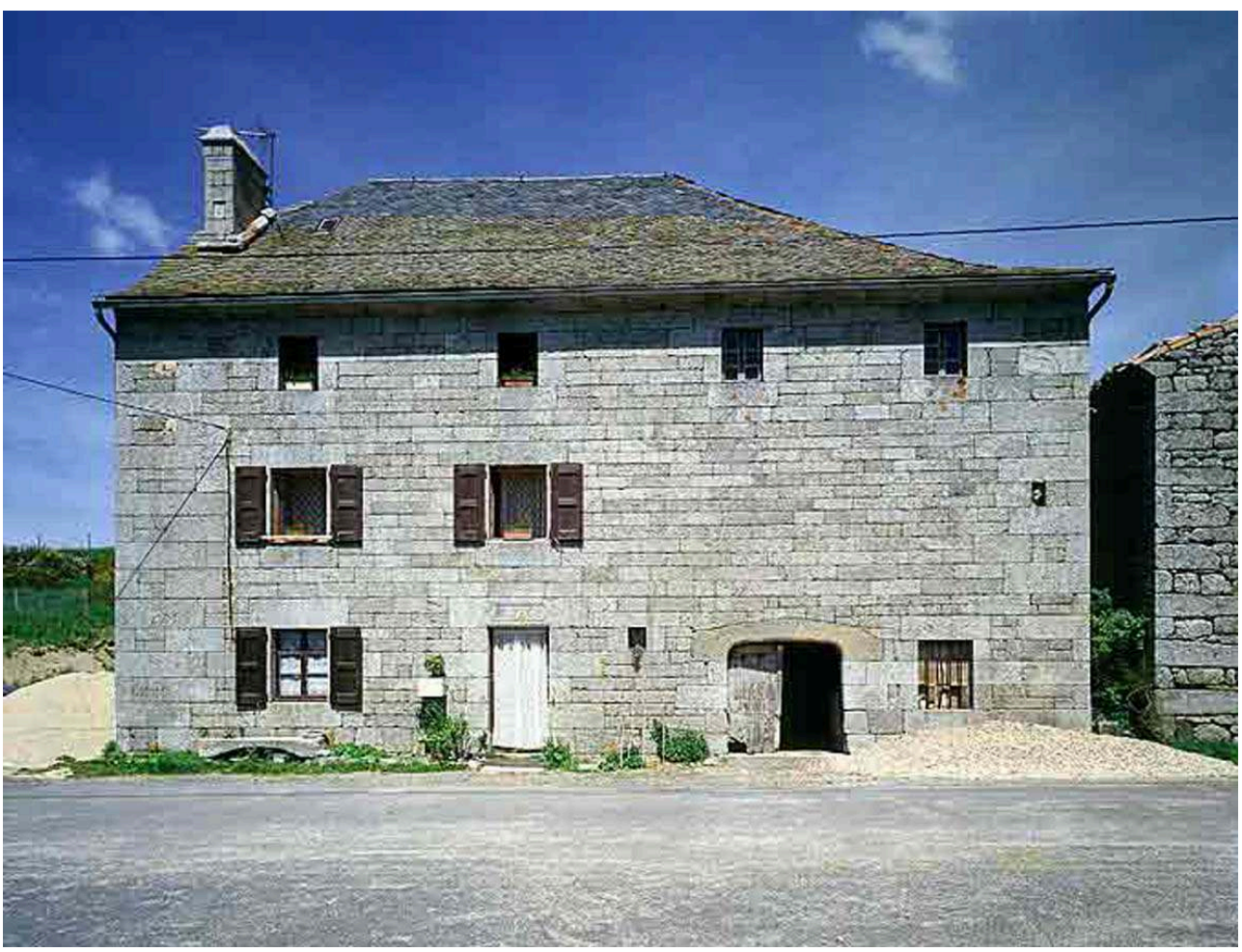

La Vachellerie (Paulhac-en-Margeride). Ferme se distinguant des précédentes par l'importance du logis qui présente un étage, l'ampleur de la grange et la qualité de la mise en œuvre. La date de 1874 est inscrite sur le linteau de la porte du logis

Phot. Inv. L. Lesur @ Inventaire général, ADAGP, 2001 
Figure 30

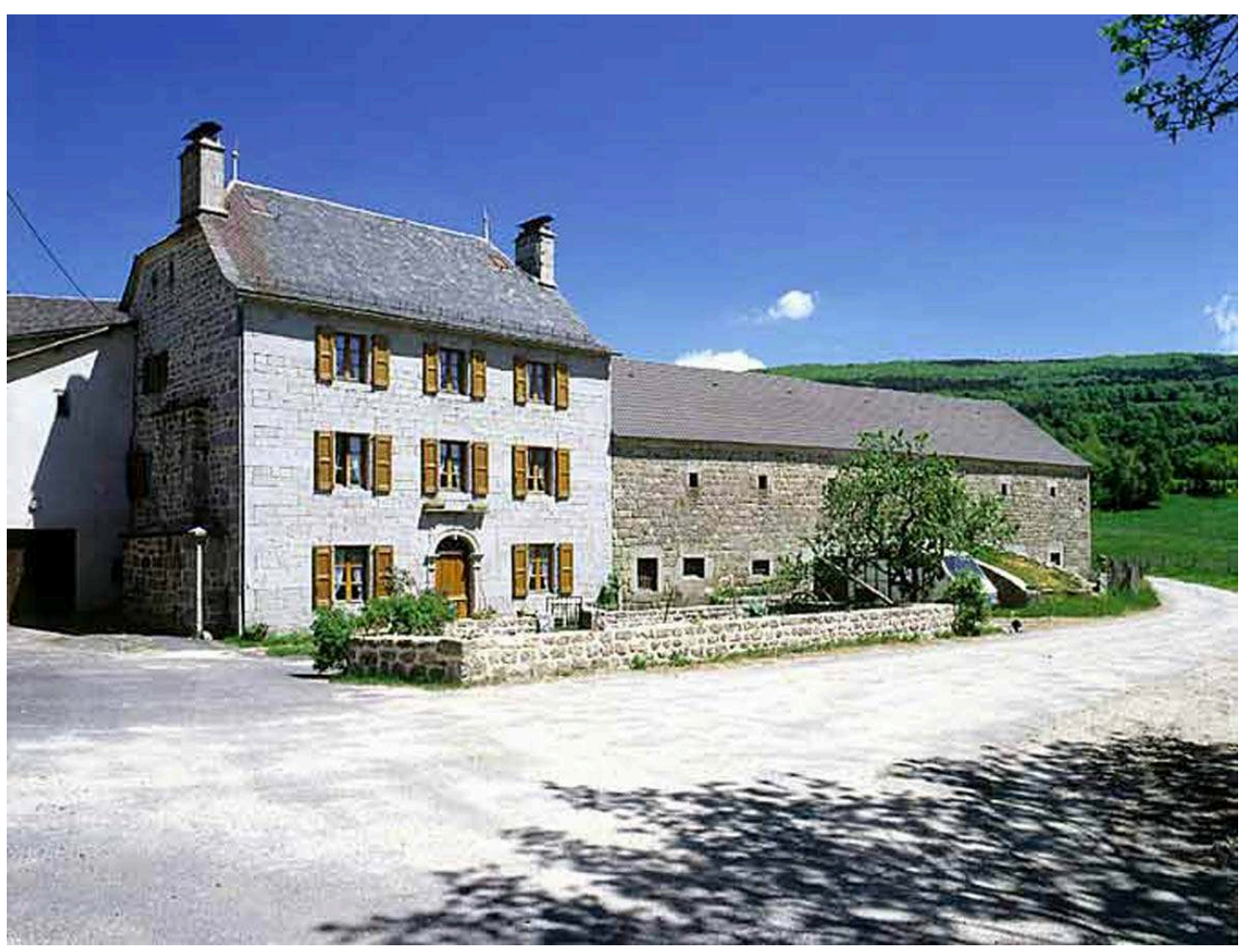

Hameau des Ducs (Le Malzieu-Forain). Ferme. Le logis et la grange-étable construits en 1896 et 1898 occupent deux corps de bâtiment contigus. Le logis a pris une importance considérable ; son élévation sur cour est ordonnancée, il est couvert d'un toit à demi-croupe. La grange-étable est peutêtre plus ancienne. La grange présente des jours d'aération réguliers, on y accède par une rampe tournante

Phot. Inv. J.M. Périn @ Inventaire général, ADAGP, 2001

La majorité des fermes sélectionnées pour étude sont situées dans les villages ou dans les écarts. On trouve cependant autour des principaux bourgs des deux cantons des fermes isolées au milieu de grands domaines comme la ferme de Taladisse sur la commune de Serverette. Mentionnée dans l'inventaire des propriétés de la famille de Moré, co-seigneur de Serverette, dressé à la Révolution, elle appartient au XIX ${ }^{e}$ siècle à une famille de notables qui possède plusieurs fermes sur les communes de Serverette et de Fontans. Le remploi dans la grange-étable partiellement reconstruite au XIX ${ }^{\mathrm{e}}$ siècle d'une pierre datée 1777, témoigne de l'existence d'un bâtiment plus ancien. Le corps de logis a peut-être été construit en 1851 (date portée sur la fontaine avec le nom du propriétaire "Blanquet Victor Guillaume "). ${ }^{17}$

\section{Les habitats temporaires, cabanes et mazets}

Pour terminer ce survol de l'architecture rurale en Languedoc, nous souhaiterions donner quelques exemples de cabanes, bâtiments annexes de la ferme ou de la maison de village. Cabanes de pêcheurs, cabanes de vigne ou mazets, cabanes de pierre sèche, habitats temporaires ou simples abris, ce sont des constructions particulièrement menacées. Cependant, elles ponctuent encore certains territoires. Les cartes I.G.N. ne les mentionnant pas systématiquement et le cadastre ne les représentant jamais, leur repérage dans le cadre d'opérations d'inventaire rapide reste aléatoire, se fondant sur 
la toponymie, l'enquête orale et les nombreux travaux réalisés par les associations ${ }^{18}$. Le quadrillage systématique qui serait nécessaire ne peut être réalisé qu'exceptionnellement et dans le cadre d'enquêtes thématiques telle que celle qui a été réalisée sur la commune de Bédarieux dans l'Hérault.

Il ne reste plus que quelques rares exemples des cabanes de pêcheurs construites en roseau dans la zone palustre du littoral, parfois sur le domaine maritime. Près d'AiguesMortes où elles ont toutes disparu, leur présence est attestée par une description de 1839 et par une gravure de Taylor et Nodier.

Elles étaient construites en pierre et roseau, ou entièrement en roseaux, sur un plan rectangulaire à abside. Les cabanes photographiées dans le cadre d'une opération d'urgence menée en 1967 dans la commune du Barcarès, appartiennent au même type ${ }^{19}$ (fig. $\mathbf{n}^{\circ}$ 31, 32).

Figure 31

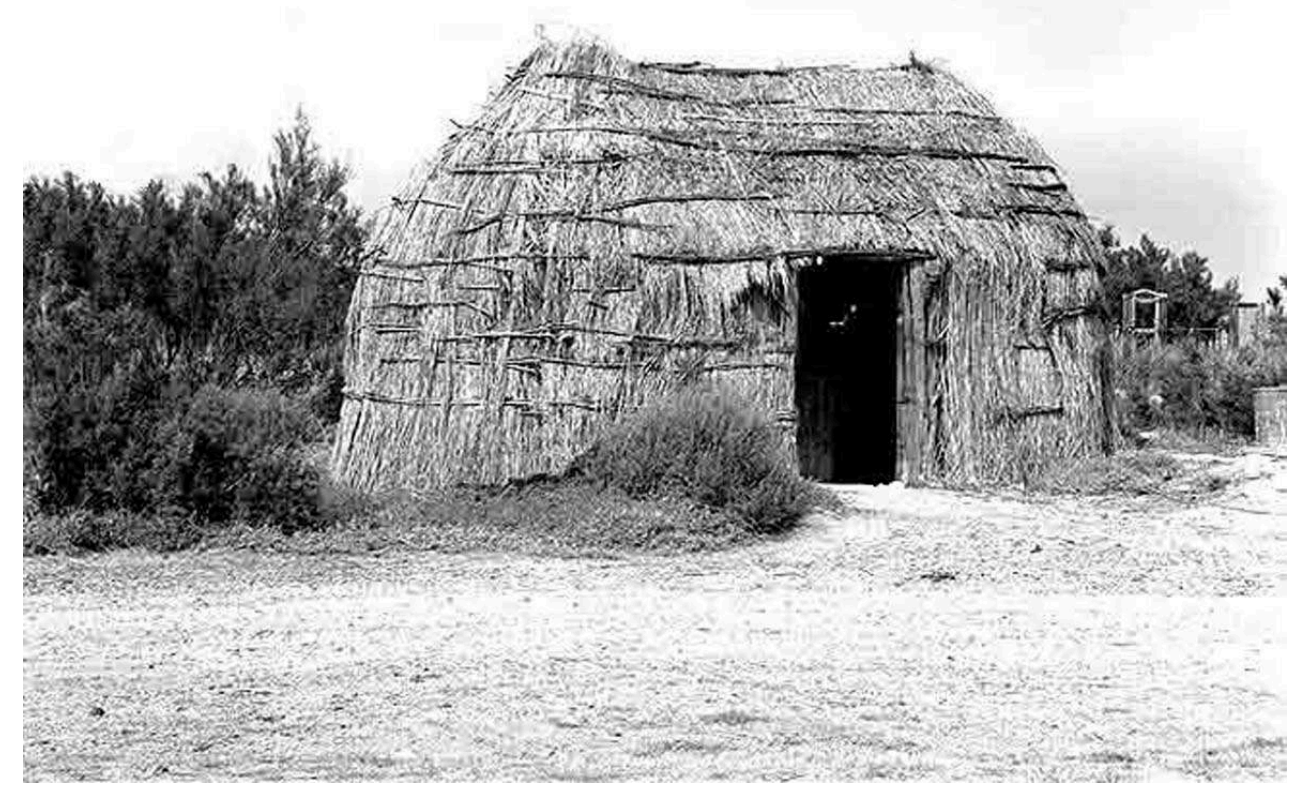

Le Barcarès. Etang de Salses. Cabane de pêcheur. Détail de cabane de plan elliptique à deux absides Phot. Inv. M. Descossy (c) Inventaire général, ADAGP, 1967. 


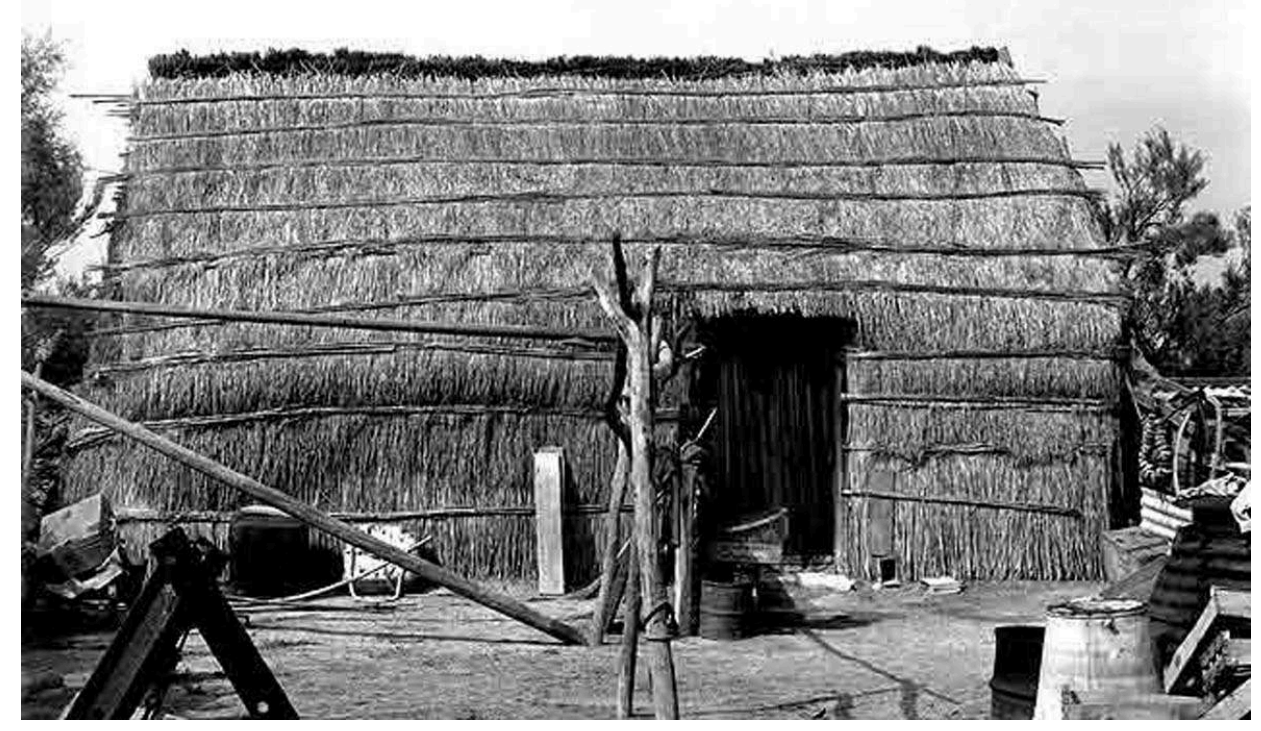

Le Barcarès. Etang de Salses. Cabane de pêcheur. Façade

Phot. Inv. M. Descossy @ Inventaire général, ADAGP, 1967

41 D'un type tout différent, les cabanes en pierre sèche sont fréquentes dans des pays de garrigues. Ce sont, comme les mazets, des abris temporaires, dépendances lointaines de la maison principale située au village. Leur construction correspond sans doute à des périodes de mise en culture de terres nouvelles par épierrement et à la culture de l'olivier. Les murs de pierre sèche qui quadrillent le territoire de certaines communes atteignent généralement une épaisseur considérable et les cabanes, souvent situées en bordure de parcelles, y sont adossées. Le plus souvent de plan circulaire, elles sont toutes construites selon la technique de la voûte en encorbellement.

42 Faite de basalte et construite en gradin, la cabane de l'Auverne (commune de Celles, canton de Clermont l'Hérault) est un exemple remarquable de ce type d'architecture, tant par sa hauteur que par le matériau utilisé. ${ }^{20}$

43 A distance des agglomérations, les cabanes de vigne ou mazets parsèment la plaine viticole. Habitat temporaire destiné à abriter les gens et les outils de travail, elles comportent parfois des cuves à vin bâties en pierre et garnies de carreaux de faïence, un puits ou une citerne (Le Pouget). Leur usage n'était cependant pas exclusivement utilitaire. Situé en pleine campagne, à l'écart du village où les espaces libres sont rares, ombragé par un pin ou environné d'arbres fruitiers, le mazet était un but de promenade dominicale.

Le travail mené sur la commune de Bédarieux montre que peuvent être associés sur un même territoire cabanes de pierre sèche et mazets. ${ }^{21}$

45 Quels vœux formuler pour la poursuite du travail dans le domaine de l'habitat rural dans cette période de mutation du service de l'Inventaire? On souhaiterait tout d'abord 
développer la connaissance de territoires tels que la plaine rhodanienne ou les montagnes et les vallées pyrénéennes afin de constituer un échantillonnage réellement représentatif à partir duquel on pourrait tirer des conclusions dépassant le cadre administratif du canton ou du département et répondre à des questions portant par exemple sur la répartition des matériaux et les techniques de gros œuvre ou de couverture, sur l'implantation, la distribution, etc.

Les inventaires réalisés, qui dans l'ensemble s'en sont tenus à l'identification des édifices, permettent de dégager des territoires ou des thèmes sur lesquels il faudrait mener des études prenant en compte les dépouillements d'archives antérieures à la Révolution. L'exploitation du compoix, cet ancêtre du cadastre conservé pour un très grand nombre de communes, ainsi que le dépouillement des minutes notariales contribueraient à renouveler enfin la connaissance historique.

En deuxième lieu, il conviendrait de prêter une attention particulière aux périphéries urbaines envahies par les lotissements, les zones industrielles ou commerciales qui côtoient encore des fermes ou des " domaines " agricoles. Il serait urgent de repérer ces témoins de la vie rurale et de les étudier dans leur rapport avec la ville.

A travers ce travail d'inventaire, nous sommes confrontés à la transformation profonde du monde rural, conséquence de la désertification des campagnes, de la pratique de l'indivision et de la modernisation nécessaire de l'agriculture. Nous observons aussi les effets de l'urbanisation de la périphérie des petites villes et des villages. Seul un petit nombre (dix-huit) de protections au titre des Monuments Historiques porte sur l'habitat rural. Encore faut-il préciser que la plupart des fermes concernées sont protégées en tant que parties constituantes de châteaux ou de domaines. Les autres édifices sont des fermes de notables qui se distinguent de façon évidente par leur qualité architecturale et historique. Pour exemple nous citerons deux édifices situés l'un dans la vallée du Lot, au pied du causse de Sauveterre et l'autre sur le Causse : le château de Ressouches (commune de Chanac) dont la ferme est un exemple remarquable de construction caussenarde, celle du Massegros doit sa notoriété au caractère monumental de sa galerie haute. ${ }^{22} 23$

\section{NOTES}

1. Inventaire général des monuments et des richesses artistiques de la France. Principes, méthode et conduite de l'Inventaire général. Dir. Michel Melot, Hélène Verdier ; réd. Xavier de Massary, Georges Coste. Paris : Editions du patrimoine, 2001. (coll. Documents et méthodes, $\mathrm{n}^{\circ} 9$ ). 2. Sous logiciel Texto.

3. Inventaire général des monuments et des richesses artistiques de la France. Commission régionale du Languedoc-Roussillon. Canton d'Aigues Mortes. Gard. Paris : Imprimerie nationale, 1973. (coll. Inventaires topographiques, $n^{\circ} 4$ ).

4. Inventaire général des monuments et des richesses artistiques de la France. Commission régionale du Languedoc-Roussillon. Canton d'Aigues Mortes. Gard. Paris : Imprimerie nationale, 1973 (coll. Inventaires topographiques, $n^{\circ} 4$ ). Voir : PICHERAL, Henri. Introduction géographique, p. 10-14. Voir aussi les Observations générales-architecture, p. 115-120. 
5. Signoles, André et Salanson, Hélène. Canton de Mauguio. Commune de Mauguio, dossier Exploitations agricoles, texte dactylographié, Service régional de l'Inventaire, Direction régionale des affaires culturelles du Languedoc-Roussillon. 1972.

6. Marty-Ferriol, Marie-Chantal. Le patrimoine rural bâti des grands domaines viticoles dans l'Aude. Mémoire dactylographié, Service régional de l'Inventaire, Direction régionale des affaires culturelles du Languedoc-Roussillon, 1994.

7. Inventaire général des monuments et des richesses artistiques de la France. Service régional du Languedoc-Roussillon. Gignac : un canton de la moyenne vallée de l'Hérault. Réd. Hélène Palouzié-Gouedar. Montpellier : Association pour la connaissance du patrimoine du LanguedocRoussillon, 1992 (coll. Images du patrimoine, $\mathrm{n}^{\circ} 104$ ).

8. Canton de Gignac. Dossier collectif Maisons-fermes, 45 pages dactylographiées, 34 planches de croquis et de plans schématiques, 96 photographies. Synthèse rédigée par Bernard Sournia en 1978 à partir des 312 dossiers établis sur le canton par l'ensemble de l'équipe.

9. Inventaire général des monuments et des richesses artistiques de la France. Service régional du Languedoc-Roussillon. Clermont-l'Hérault et son canton (Hérault). Dir. Francine Arnal ; réd. Marie-Sylvie Grandjouan. Montpellier : Association pour la connaissance du patrimoine du Languedoc-Roussillon, 1988 (coll. Images du patrimoine, $\mathrm{n}^{\circ} 33$ ).

10. Fabre, Ghislaine. La seigneurie : impact topographique, enceintes urbaines et châteaux-forts de Montpellier (1140-1206). Congrès national des Sociétés savantes. Clermont-Ferrand, 1992, p. 399-435. Baudreu, Dominique et Fabre, Ghislaine. La formation des villages en Languedoc. Un urbanisme diversifié. Revue des Monuments Historiques, n 187, mai-juin 1993.

11. Canton de Gignac : dossiers “Village ”, D.R.A.C. Languedoc-Roussillon. Notices versées dans la base Mérimée du ministère de la Culture et de la Communication.

12. Jourdan, Geneviève et Signoles, André. Architecture rurale du Larzac méridional. Connaissance du pays d'Oc, $\mathrm{n}^{\circ} 37,1979$, p. 21-28. Synthèse des observations effectuées sur le terrain au cours du préinventaire du canton du Caylar.

13. Canton de Villefort. Notices et images à consulter dans la base Mérimée du ministère de la Culture et de la Communication. Inventaire général des monuments et des richesses artistiques de la France. Service régional du Languedoc-Roussillon. Canton de Villefort, 1989 (coll. Images du Patrimoine, $n^{\circ}$ 63).

14. Les inventaires des cantons de La Canourgue, du Massegros, et de Chanac ont été intégrés dans la base Mérimée ; les notices sont illustrées d'images. Inventaire général des monuments et des richesses artistiques de la France. Service régional du Languedoc-Roussillon. Cantons de La Canourgue, Chanac, Le Massegros (Lozère). Montpellier : Association pour la connaissance du patrimoine du Languedoc-Roussillon, 1995 (coll. Images du Patrimoine, $\mathrm{n}^{\circ}$ 142).

15. Marres, Paul. Les grands causses, étude de géographie physique et humaine. Tours : Arrault et Cie, 1935.

16. Inventaire des cantons de Saint-Alban-sur-Limagnole et du Malzieu-Ville, notices collectives fermes, Direction régionale des affaires culturelles du Languedoc-Roussillon.

17. Voir notice IA48000961, dans la base Mérimée.

18. Voir le site du C.E.R.A.V. (Centre d'étude de l'architecture vernaculaire sous la direction de Christian Lassure) qui rassemble de nombreuses études sur ces questions, notamment sur les constructions en pierre sèche, et propose une importante bibliographie. http : www.cerav.com et www.pierreseche.com. On peut voir aussi www.cevennes-parcnational.fr.

19. Voir notice IA00029719, dans la base Mérimée.

20. Voir notice IA00028370, dans la base Mérimée.

21. Voir notice IA34000611, dans la base Mérimée.

22. Voir notice IA00133655 et PA00103811 (Chanac: château de Ressouches) dans la base Mérimée.

23. Voir notice IA48000301 et PA48000001 (Le Massegros : ferme nº 1) dans la base Mérimée. 


\section{RÉSUMÉS}

Vingt cantons ruraux ont été traités par l'équipe de l'Inventaire en Languedoc-Roussillon. Les exemples présentés ici illustrent la variété des territoires de la région : mas de la plaine littorale avant et après l'expansion de la viticulture à fin du XIX ${ }^{\mathrm{e}}$ siècle ; maisons et villages de la vallée de l'Hérault; fermes groupées en hameaux ou villages de la moyenne montagne qui couvre le nord du département de l'Hérault et la totalité du département de la Lozère où se dressent encore des édicules (fours à pain, travail à ferrer...), propriétés des communautés d'habitants; habitat caussenard du Larzac méridional et du Sauveterre lié à la pratique de l'élevage ovin; fermes bâties en terrasses dans la partie orientale des Cévennes; enfin l'exemple de la Margeride tout au nord de la région.

Twenty rural districts have been surveyed by the research workers of the Languedoc-Roussillon Inventaire. Examples shown here illustrate the wide range of territories, from the " mas » in the coastal plain, built before and after the wine-growing expansion era at the end of the 19th century, to the houses and villages in the Hérault valley. In the lower mountains covering the north of the Hérault and Lozère departments, the farms are concentrated in villages or hamlets where facilities shared by the inhabitants (bread-ovens, horse-shoeing devices...) can still be seen. Distinct also are the dwellings in the Causses, south of Larzac and Sauveterre, connected with sheepfarming, farms built in terraces in the Eastern Cevennes and the Margeride, in the northern boundaries of the region.

\section{INDEX}

Mots-clés : Larzac, Cévennes, Margeride, ferme, bergerie, mas, mazet, cabane, Hérault, Lozère, Sauveterre

\section{AUTEUR}

\section{MARIE-SYLVIE GRANDJOUAN}

Conservatrice du patrimoine, Service régional de l'Inventaire, Direction régionale des affaires culturelles du Languedoc-Roussillon. marie-sylvie.grandjouan@culture.gouv.fr 